\title{
XXV. \\ Bau und Histiogenese des pathologischen Neuroglia-Gewebes.
}

(Aus dem Pathologischen Institut der Königl. Universität Padua. Vorstand:

Prof. Dr. A. Bonome.)

Von

Prof. Dr. A. Bonome.

(Hierzu Taf. XIII-XV.)

\section{I.}

Die Untersuchungen über die Structur-Eigenschaften der Neuroglia haben in diesen letzten Jahren wegen der Anwendung einiger neuen technischen Färbungsmethoden bedeutende Fortschritte gemacht. Trotz der Lücken, die noch auszufüllen sind, namentlich bezüglich der Histiogenese der Neuroglia, kann man nicht verkennen, dass die von Weigert ${ }^{1}$ ) empfohlene Färbungsmethode sich ganz hervorragend bewährt, besonders, weil sie uns Helligkeit der Bilder und damit sehr leicht die Möglichkeit verschafft, nicht nur die Zellen des Stützapparates der Nerven- oder Ganglienzellen zu unterscheiden, sondern auch die zartesten Fäserchen deutlich zu machen und ihre Verhältnissse zu den Neuroglia-Zellen zu zeigen.

Eine solche Methode aber, obgleich sie ein gutes, technisches Hülfsmittel für das Studium der Structur-Eigenschaften des erwachsenen und normalen Neuroglia-Gewebes des Menschen darstellt, und obgleich sie hilft, gewisse Unterschieds-Charaktere zwischen den Ganglienzellen und den Deiters'schen Zellen, und ferner zwischen den letzteren und dem sie umgebenden Fäserchen-Netze festzustellen, giebt uns keine Sicherheit im Hinblick auf ilre Specificität: nehmlich bei einigen pathologischen Zuständen färbt sie in gleicher Weise die Neuroglia und gewisse Substanzen oder Elemente, wie die Fibrinfasern, die binde-

1) C. Weigert: Beiträge zur Kenntniss der normalen menschlichen Neuroglia. Frankfurt a. M. 1895. 
Virchow's Archiv. Bd. CLXIII. 1

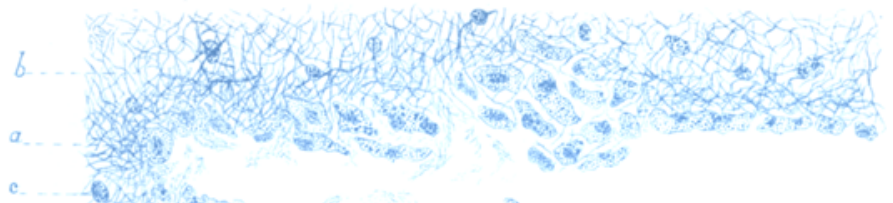

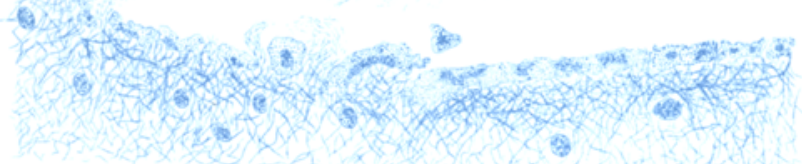

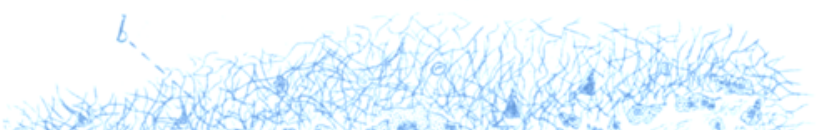

2

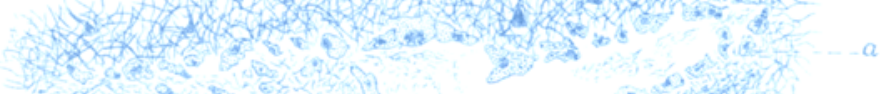
coloces
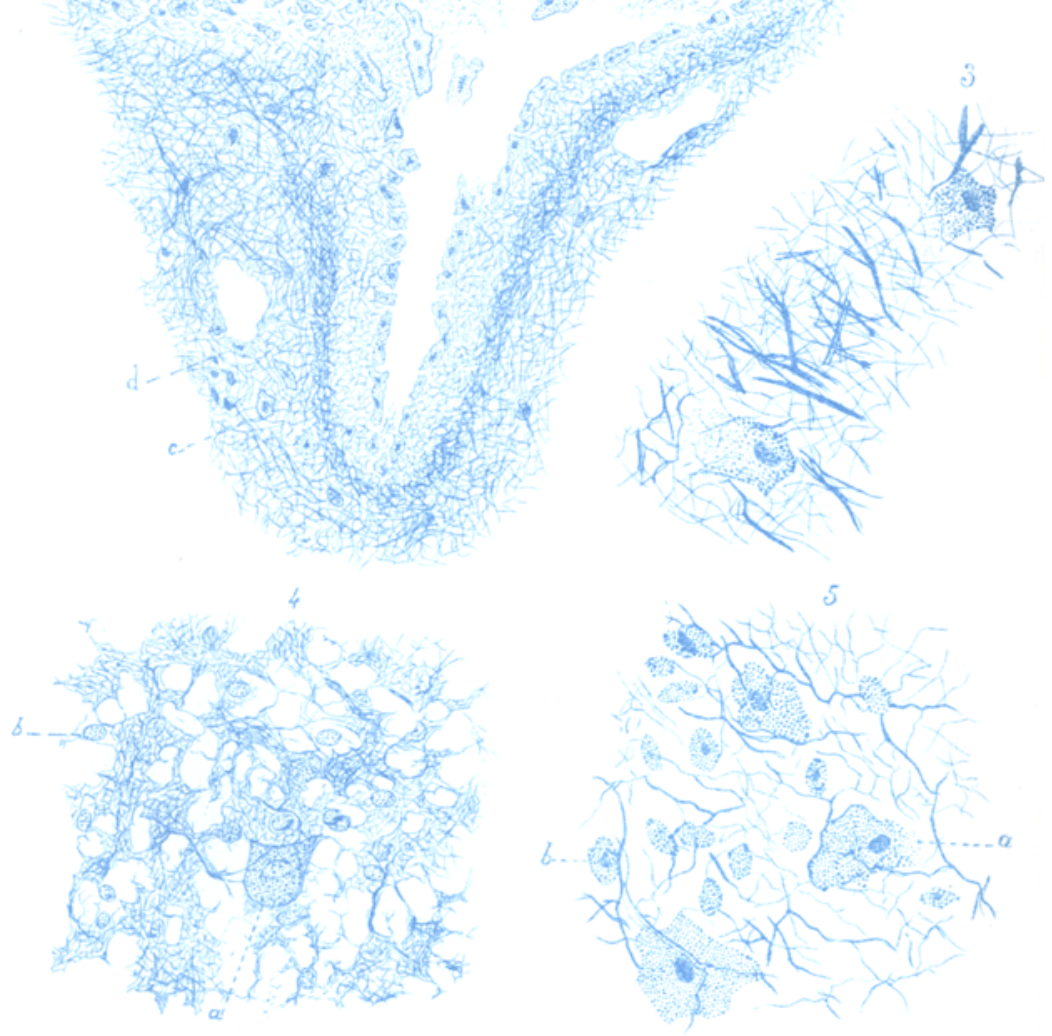
Virchow's Archiv. Bd. CLXIII.

Tafel XIV.
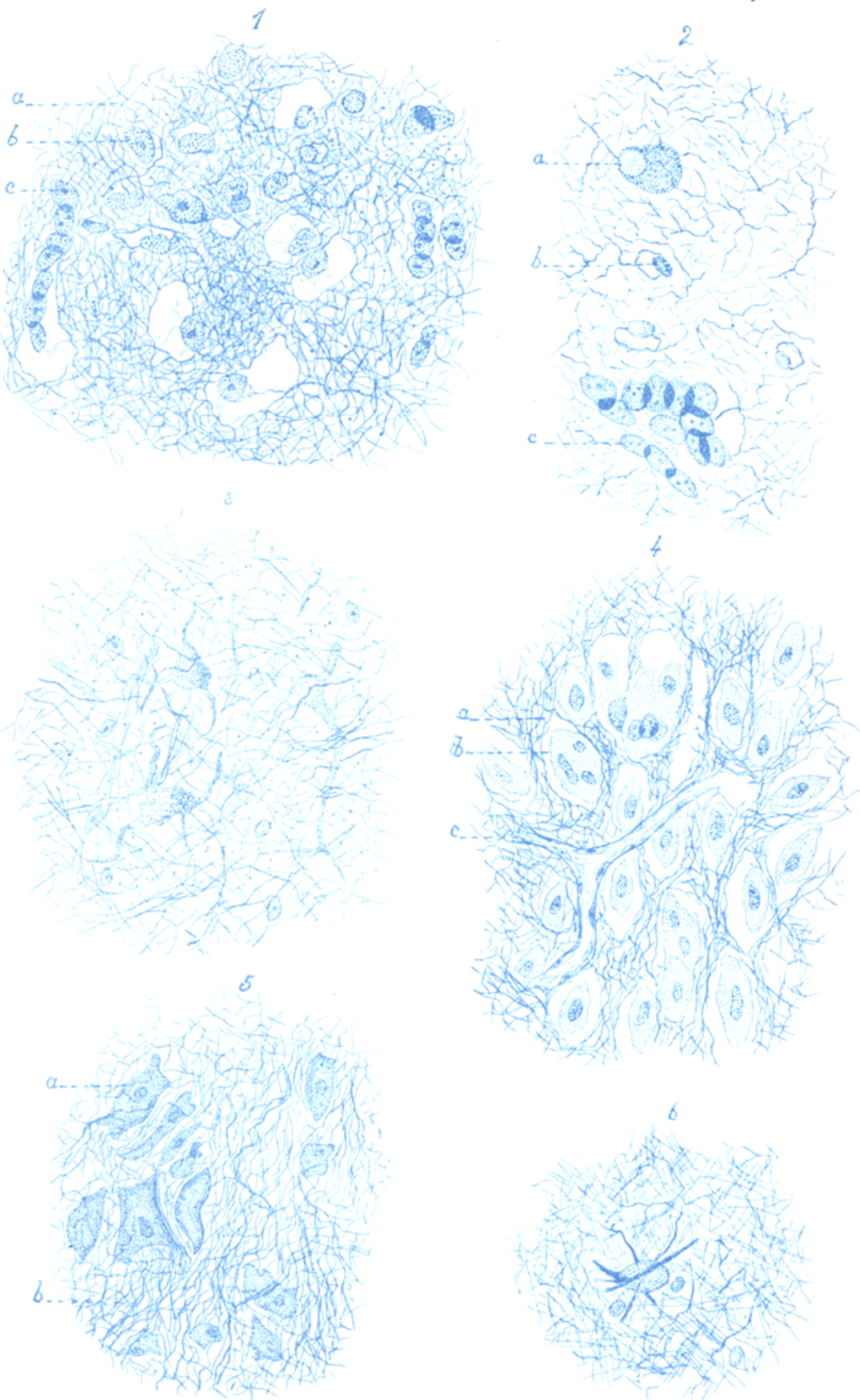


\section{2}

Y)

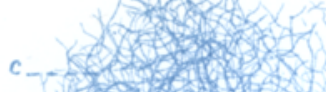

ioneres:

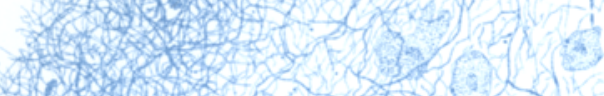

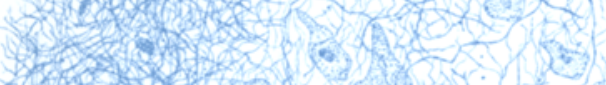
-200 a

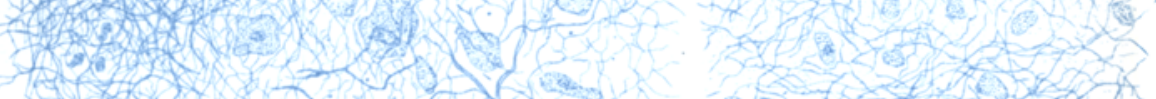
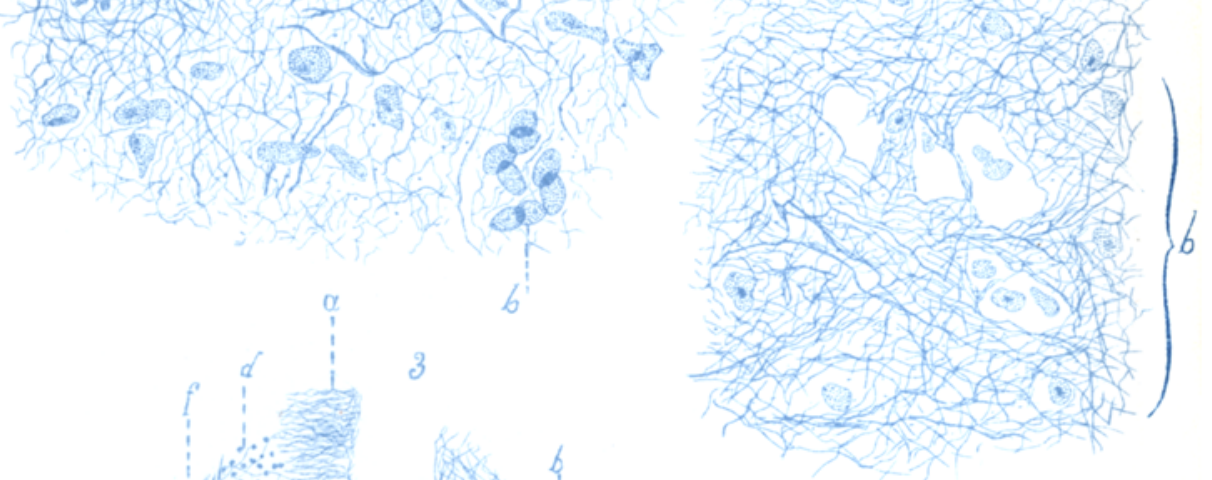

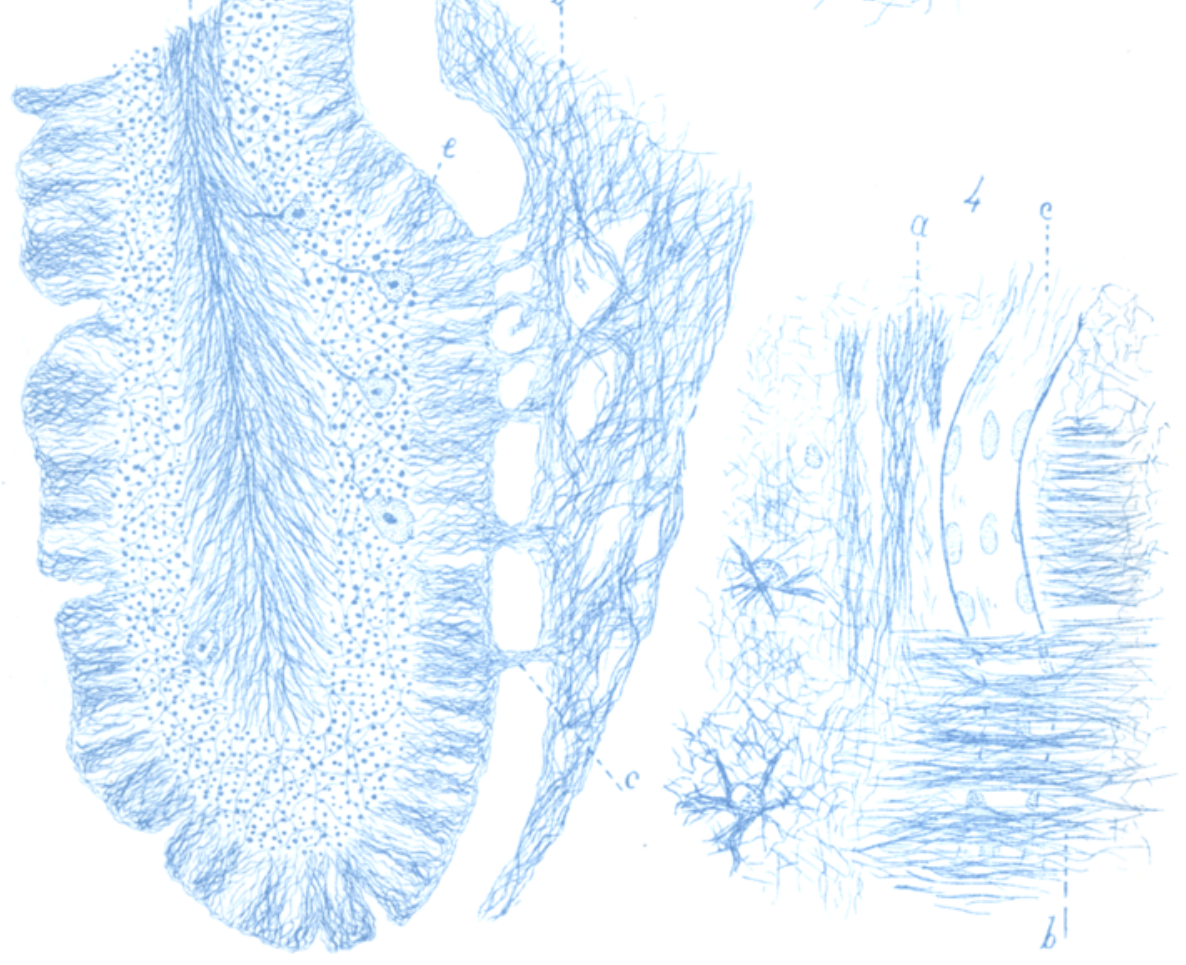


gewebigen Fasern, die Endothel-Elemente, die Blutkörperchen, welche man übrigens alle von den Theilen, aus denen die Neuroglia sich zusammensetzt, durch andere Charaktere unterscheiden kann.

Mit Benutzung seiner Methode hat Weigert die Structur der normalen menschlichen Neuroglia eingehend studirt, und in seiner Monographie hat er in belehrender: Weise die Fäserchen in ihren Verhältnissen zu den Neurogliazellen, zu anderen Substanzen und dem Bindegewebe beschrieben; einige wichtige histologische und histogenetische Eigenschaften der Neuroglia hat er selbst discutirt. Er hat die zuerst von Boll ${ }^{1}$ ) und von Ranvier ${ }^{2}$ ) ausgesproschene Meinung bekräftigt und zum grossen Theil als richtig bewiesen, nach welcher die einst als Fortsätze der Deiters'schen Zellen betrachteten Neuroglia-Fäserchen dem Protoplasma chemisch nicht gleichen, sondern sich von demselben durchaus unterscheiden. Eine solche Differenzirung würde sich zeigen nicht nur bei den in einer gewissen Entfernung vom Zellkörper gelegenen Fäserchen, so dass man vermuthen könnte, dass sie von Veränderungen abhängig wäre, welche stattfinden, je nachdem die Fäserchen sich aus dem Zellkörper entfernen, sondern im Gegentheil würde sie auch ganz in der Nähe des Zellkernes erscheinen.

Nach Weigert würden bei Anwendung seiner Färbungsmethode Fortsätze bei den Zellen der normalen Menschen-Neuroglia fehlen, oder mindestens wären solche Fortsätze auch im farblosen Zustande nicht sichtbar. Das spinnenförmige oder astrocytenförmige Aussehen dieser Zellen würde davon herrühren, dass die Neuroglia-Fäserchen, nachdem sie an mehreren Stellen in den zelligen Körper hineingedrungen sind, aus der entgegengesetzten Seite oder aus, den Eingangsorten nahe gelegenen Punkten austreten, während sie sich vom zelligen, durchsichtigen und farblosen Protoplasma immer different erhalten.

1) Boll: Die Histologie und Histiogenese der nervösen Centralorgane. Archiv für Psychiatrie Bd. IV 1874.

2) Ranvier: De la névoglie, Compt. rendu de l'Acad. des Sciences, p. 921882 .

Ranvier: De la névoglie, Archives de Physiologie normale et path. 1883. 
Dieser Mangel an wirklichen Fortsätzen in den Neurogliazellen bei den nach der Weigert'schen Methode vorbereiteten Präparaten würde, der Meinung von Weigert nach, eine der wichtigsten und sichersten Normen abgeben, um die Zellen des Stützapparates von den ächten Ganglienzellen zu unterscheiden, welche, wie bekannt, dendritische und Cylinderachsen-Fortsätze besitzen.

Das Verhältniss zwischen dem Netze der nach solcher Methode färbbaren Fäserchen (Neuroglia-Fäserchen) und dem Körper der Neurogliazellen würde den Unterschied zwischen den beiden Zellenarten noch deutlicher machen. Da nun die Erfahrung uns zeigt, dass das ganze wirklich nervöse Material sich bei Anwendung der Weigert'schen Methode nicht färbt, müssen wir wohl schliessen, dass jene Fäserchen, welche sich blauschwarz färben, nicht nervöser Natur seien.

Andererseits müssen wir gerade deshalb, weil solche Fäserchen sich bei der erwähnten Methode stark blau färben, während die protoplasmatische Substanz farblos bleibt, annehmen, dass diese Fasern aus einer vom Protoplasma der Neuroglia-Zellen verschiedenen und von dem zelligen Körper emancipirten Sub$\operatorname{stanz}$ bestehen.

Auf Grund dieser Unterscheidbarkeit der Fäserchen hat Weigert die entschiedene Meinung ausgesprochen, dass das von den Fäserchen gebildete Netz nicht als eine unmittelbare Emanation des Körpers der Neuroglia-Zellen, sondern als eine ächte und wirkliche Intercellular-Substanz zu betrachten sei. -

Die von Weigert aufgestellten Thatsachen bestätigen nicht nur die Hypothese von Ranvier, sondern sie beweisen auch, dass die verschiedenen Methoden der schwarzen Reaction, besonders diejenige der Versilberung von Stückchen, die mit Chromsalzen gehärtet wurden (die classische Golgi'sche Methode), uns nicht hinreichende Thatsachen über die Structur der Neuroglia-Zellen verschaffen, so dass es nicht möglich ist, die Differenzirung der Fäserchen in dem zelligen Körper zu beobachten und die nervösen Zellen von den Neuroglia-Zellen leicht und sicher zu unterscheiden. Diese Unterscheidbarkeit gründet sich bei den nach Golgi'scher Methode hergestellten Präparaten auf die Form-Eigenschaften der schwarzen Silhouette der Zelle 
und gar nicht auf die Charaktere einer färbenden Reaction, so dass bloss sehr gut geübte Personen eine Unterscheidung treffen können und dass es manchmal auch für diese schwer und unsicher wird. -

Wenn nun auch die wichtigen, von Weigert mit seiner Methode gefundenen Structur-Einzelheiten der Neurogliazellen wirklich im Stande sind, den alten Begriff der Astrocyten so zu verändern, dass sie als Zellen ohne protoplasmatische Fortsätze erscheinen können, so widersprechen sie doch nicht der Hypothese, nach welcher der Zellkörper; indem er sich mehr oder weniger tief an einigen Stellen modificirt, zur Erzeugung der bekannten fibrillären Bildungen in irgend einer Weise mitwirken kann.

Diese Hypothese, die Weigert bezüglich der normalen Neuroglia nicht erörtert hat, halte ich für wahrscheinlich im Falle der pathologischen Neuroglia-Bildung.

Ich habe in der That einige Befunde bemerkt, die ich weiter unten berichten werde, aus welchen sich zu ergeben scheint, dass auch das Protoplasma der Neurogliazellen zur Bildung der Fäserchen mitwirke. Damit wäre die Hypothese von $\mathrm{K}_{0}$ elliker ${ }^{1}$ ) wieder aufgestellt, nach welcher das Golgi'sche Zellen-Protoplasma aus zwei Theilen bestehen würde: aus einem centralen und aus einem peripherischen, welcher letztere, indem er sich chemisch verändert, die Fortsätze bilden würde, nachdem er sich von dem centralen, den Kern enthaltenden Theile getrennt hat. - Wenn man annimmt, wie ich es durch meine Präparate beweisen kann, dass die peripherische Protoplasma-Schicht sich bei gewissen pathologischen Neuroglia-Zellen so verändern kann, dass sie genau wie die Fäserchen reagirt, so hätten wî̀ den Begriff von Weigert theilweise $z u$ modificiren, nach welchem die Neuroglia-Zellen nur Unterbrechungspunkte des Neuroglia-Netzes darstellen würden, neben welchen die Fäserchen sich nur in Berührung mit den Zellen finden.

Andererseits steht diese Entstehungs-Art derFäserchen, welche sich mit den Neurogliazellen sehr reichlich durchflechten, so.

1) Koelliker: Handbuch der Gewebelehre des Menschen, II, Bd. 1. H. S. 150 . 
dass sie eine gewisse Grundsubstanz bilden, nicht mit der Meinung. Weigert's in Widerspruch, und näbert das Neuroglia-Gewebe dem Bindegewebe; denn es ist bekannt, dass das zellige Protoplasma bei einigen Formen des letzteren das Material für die Bildung der Grundsubstanz liefert.

Obgleich noch nicht alle die Neuroglia-Histiogenese betreffenden Fragen, besonders vom vergleichenden embryologischen Standpunkte aus, gelöst worden sind, nimmt der grösste Theil der Histologen heutezutage an, dass die Neuroglia von ausschliesslich ektoblastischer Natur sei, und dass die Zellen derselben, obwohl sie durch ibre morphologischen Eigenschaften nicht mehr an die Epithelzellen der ursprünglichen nervösen Schicht, aus der sie entstanden sind, erinnern, mit Elementen mesoblastischen Ursprungs nicht $z u$ verwechseln sind, deren Theilnahme an der Bildung der Neurogliazellen ausgeschlossen wird. Diese Neurogliazellen wären also nichts anderes, als modificirte Epithelzellen, welche mit den Fäserchen zur Bildung eines Stützapparates für die Nervensubstanz mitwirken, und deshalb verhalten sie sich wie ein Bindegewebe.

Die bis jetzt gemachten Beobachtungen über die pathologische Entwicklung der Neuroglia würden diese Auffassung bestätigen. Solche Beobachtungen wurden aber mit technischen Methoden ausgeführt, welche wenig geeignet waren, die Fäserchen, deutlich zu machen und sie nicht nur innerhalb des Protoplasma der Stützzellen, sondern auch von einigen pathologischen Producten, z. B. von Fibrin, von den Fasern des Bindegewebes u. s. w zu unterscheiden.

Um die Entstehungs-Einzelheiten dieser pathologischen Neuroglia zu erforschen, die Verhältnisse zwischen den Fäserchen und den neugebildeten Zellen festzusetzen, und die Art und Weise zu studiren, wie dieselbe sich zu den nervösen Elementen einerseits und zu dem Bindegewebe andererseits verhält, habe ich seit ein paar Jahren eine Reihe von Versuchen unternommen an allen Glia-Neubildungen, die ich in den nervösen Centren von menschlichen Leichnamen auffinden konnte.

Solche Neubildungen zeigten sich bald an den Gehirnwindungen, bald in der unterliegenden weissen Substanz, bald im Centrum Semi ovale wieussenii oder in dem Commissuren- 
System, bald endlich in den Ganglien der Basis und in dem verlängerten Mark.

Schon nach der makroskopischen Untersuchung dieser Neubildungen konnte man die wirkliche Theilnahme der Pia mater und der Arachnoides ausschliessen, und es war deshalb sicher, dass es in diesem Fall sich nicht um sarcomatöse, aus den Hirnhäuten entstehende Neubildungen handelte. -

Einige von diesen Neubildungen besassen die histologisehen Eigenschaften der Neuroglia-Hyperplasie, ohne dass man eine grosse Atypie an ihren zelligen Elementen bemerkte, welche übrigens verhältnissmässig nicht sehr zahlreich waren, so dass sie die Charaktere einer Gliose darstellten.

Andere dagegen, welche die Hirnwindungen und namentlich die unterliegende markhaltige Substanz betrafen, zeigten in deutlicher Weise den gliomatösen Typus, sie hatten aber eine beträchtliche Zahl von zelligen Elementen, die in Beziehung auf die Form, auf die Grösse und auf ihr Verhältniss zu den Fäserchen, absolut nichts Gleichartiges mit den Zellen der normalen Neuroglia hatten. Andere dieser Neubildungen erregten ein nicht gewöhnliches Interesse, da man im Inneren derselben Zellen von epithelialem Aussehen beobachtete, die an die ectodermischen Zellen der primitiven nervösen Schicht erinnerten.

Um kurz zu sein, halte ich es für angezeigt, den nekroskopischen Befund jedes einzelnen von mir untersuchten Falles und auch die makroskopische Beschreibung der neun von mir beobachteten gliomatösen Neubildungen zu unterlassen. Ich werde mich bloss auf die Beschreibung der Einzelheiten beschränken, welche die wichtigsten Fragen nach der Glia-Neubildung bei pathologischen Zuständen betreffen können.

II.

Angeborene und erworbene Neubildungen des Neuroglia-Gewebes. Gliome und Gliose.

Die erste Frage, die uns entgegentritt, ist die, ob man die Neuroglia-Neubildung als die Folge von Entwicklungs-Störungen an den Nervencentren in den meisten Fällen betrachten kann, und ob anatomische, angeborene Dispositionen vorhanden sind, um die Entstehung gewisser Gliome zu erklären, wie es Cohnheim 
bei der Entwicklung anderer Geschwülste angenommen hat. Einige Gliom-Typen zeigen solche Eigenschaften und sind mit Entwicklungs-Störungen der nervösen Centren so innerlich verbunden (Spina bifida, Meningo-Myelocele, Syringomyelie, Encephalocele, Rückenmarks-Verdoppelung), dass die Hypothese ihrer embryonalen oder fötalen Entstehung möglich wird.

Solche sind: der von Hartdegen ${ }^{1}$ ) beschriebene Fall (bei einem Neugeborenen mit Spina bifida und mit Geschwülsten im Inneren der lateralen Hirnventrikel), der von Baumann ${ }^{2}$ ) berichtete Fall bei einem Kinde und die in einer Arbeit von Hildebrand ${ }^{3}$ ) gesammelten Fälle, ferner der interessante, von Stroebe ${ }^{4}$ ) beschriebene Fall eines bei einem 62jährigen Manne vorhandenen Glioms des occipitalen und parietalen linken Lappens, welches von Epithel ausgekleidete Cysten enthielt, und dazu die von Reymond ${ }^{5}$ ) in seiner Arbeit zusammengestellten Fälle, welche sich auf Beobachtungen von Lanceraux, Hayem, Lesage und Legrand, von Virchow ${ }^{6}$ ), von Mosler ${ }^{7}$ ), von Simon ${ }^{8}$ ), von Lachmann ${ }^{9}$ ), von Miura ${ }^{10}$ ) u. A. beziehen.

Es giebt aber keinen sicheren Beweis, um diese Hypothese immer bekräftigen zu können.

In der That ist der Befund epithelialer, denjenigen des primitiven Neuro-Epithels ähnlicher Bildungen an der Gliom-Decke

1) Hartdegen, Ein Fall von multipler Verhärtung des Grosshirns v. s. w., Archiv f. Psychiatrie Bd. 11, 1881.

2) Baumann, Zur Kenntniss der Gliome und Neurogliome. Ziegler's Beiträge Bd. VII, 1888.

3) Hildebrand, Patholog.-anatom. und klin. Untersuchungen zur Lehre der Spina bifida $u$. der Hirnbrüche, Deutscbe Zeitschr. f. klin. Chirurgie Bd. 36, 1893.

4) Stroebe, Ueber Entstehung und Bau der Gehirngliome, Ziegler's Beiträge Bd. XVIII H. III.

5) Reymond, Contribution à l'étude des tumeurs du cerveau. - Un cas de Gliome neuroformative, Archives de Neurologie p. XXVI 1893.

6) Virchow, Die krankbaften Geschwülste, Bd. II.

7) Mosler, Dieses Archiv Bd. 43 .

8) Simon, Die Spinnenzellen- und Pinselzellen-Gliome, Dieses Arcbiv Bd. 61 .

9) Lachmann, Archiv f. Psychiatrie Bd. XIII S. 50, 1882.

10) Miura, Ueber Gliome des Rückenmarkes und Syringomyelie, Ziegler's Beiträge Bd. XI, 1891. 
nicht immer eine genügende Gewähr, um die embryonale Geschwulst-Entstehung annehmen zu können.

Auf nicht unbedeutende Schwierigkeiten und Unsicherheiten stösst man, wenn man die Entstehung der bei Erwachsenen oder bei älteren Personen auftretenden Gliome erklären will. -

Wenn es in solchen Fällen auch nicht ganz scheint, die Hypothese einer Veränderung der ersten Bildung anzunehmen, so darf man doch nicht auf die Möglichkeit verzichten, dass die Glia-Elemente zu einem Zustande zurückkehren, in welchem bestimmte Eigenschaften des embryonalen Lebens zum Ausdruck kommen. Solche Rückkehr zu dem Embryonal-Zustand ist, meiner Meinung nach, eine der wichtigsten Bedingungen ihrer Vermehrung. Damit ein solcher Zustand entstehe, ist es garnicht nothwendig, dass Zersetzungs- und Zerstörungs-Vorgänge in der nervösen Substanz vorausgehen; denn man bemerkt oft keine echte Neuroglia-Proliferation um Heerde ähnlicher Natur, wie um Tuberkelknötchen, oder im Inneren der eben enstandenen Entzündungsheerde.

Wir können also sagen, dass die Bedingungen, welche bei pathologischen Zuständen geneigt sind, die Neuroglia-Proliferation zu erzeugen, uns sehr wenig bekannt sind.

Jedenfalls darf man wohl annehmen, dass solche anomale Proliferation, sei es während der Entwicklung der nervösen Centren, sei es später, wennn dieselben ihre vollständige Ent wicklung erreicht haben, stattfinden kann.

Und da man je nach der Entwickelungs-Art dieser abnormen Proliferationen der Neuroglia Unterschiede bemerken kann, sei es bezüglich der Zahl der zelligen Elemente, ihrer Form, ihrer Vereinigungs-Art und ihres Verhältnisses zu dem Fäserchensystem, sei es bezüglich des Verhaltens der nervösen Fasern, der Ganglienzellen, und auch der Blutgefässe und der Hirnhäute, so schien es mir möglich, auf Grund der durch die Untersuchung von zahlreichen pathologischen Stücken in den letzten Jahren zusammengestellte Resnultate die ächten Gliome von den einfachen Gliosen mit Hülfe von objectiven und rationellen Kriterien zu unterscheiden. -

Zuerst kann man bestimmt sagen, dass die Gliome ächte Geschwülste im Allgemeinen darstellen, welche, wenn sie auch 
nicht immer scharf begrenzt sind, dennoch immer eine gewisse Begrenzung haben, während die einfachen Gliosen die wichtigste Eigenschaft der Geschwulst, (id est die Anschwellung und die Begrenzung), gewöhnlich nicht zeigen.

Zweitens sind die Gliome im Allgemeinen an zelligen Elementen reicher, als die einfachen Neuroglia-Hyperplasien, sodass es manchmal sehr schwierig ist, dieselben von einigen Sarcom-Formen (Endothel-Sarcomen) zu differenziren; dieselben sind auch mit im Ueberfluss neugebildeten Blutgefässen versehen, welche ektasisch sind und dünne Wände haben, so dass sie leicht zerreissen und Haemorrhagien erzeugen. Dadurch erscheinen die ächten Gliome bei einer makroskopischen Untersuchung als weiche Anschwellungen von grau-rosenfarbigem Aussehen, mit roth-schwarzen Pünktchen oder auch mit schwärzlichen Zonen, die verhältnissmässig ausgedehnten Gefässen und kleinen Haemorrhagien entsprechen. Die Gliosen dagegen zeigen sich als Verdichtungen und ausgebreitete, blass grau gefärbte Verhärtungen.

Wegen ihrer zahlreichen Zellen und Gefässe gehen die Gliome regressiven Metamorphosen entgegen. Sie werden theilweise weich und erleiden eine Nekrubiose, und in Folge der Resorption der weicher gewordenen Theile entstehen Höhlen, die mit weichen, gelblichen, halbdurchsichtigen und unregelmässigen Wänden versehen sind, innerhalb deren man blutige Klümpchen oder Reste von ergossenem Blute finden kann. Dass ist bei den einfachen Gliosen nicht bemerkbar. Ausserdem findet man bei den Gliomen, abgesehen von einigen seltenen angeborenen Formen, im Allgemeinen keine Nervenfasern und Ganglienzellen, während das bei den Gliosen stattfinden kann; es ist mir möglich, dies durch einen typischen Fall von ausgedehnter Gliose der Corpora quadrigemina bei einem 12 Jahre alten Mädchen zu bestätigen.

Die zelligen Gliom-Elemente unterscheiden sich, wie ich weiter in ausführlicher Weise zeigen werde, sehr von den Zellen sowohl der normalen ausgebildeten, als auch der in der Entwicklung stehenden Glia.

Die Proliferation derselben ist die Folge einer tiefen Ernährungs-Störung, derenwegen die ächten nervösen Elemente 
nicht mehr wachsen und sich zersetzen, während die Gliazellen ihre embryonalen Eigenschaften wieder aufnehmen und sich. mehr oder weniger schnell vermehren.

Einige derselben, welche eine starke proliferirende Thätigkeit nicht besitzen, nehmen morphologische Charaktere an, welche den Eatoderm-Elementen gleichen, während andere, die eine stärkere produetive Thätigkeit haben, sich wie die embryonalen, Sarcome bildenden Zellen verhalten.

Das Fäserchen-System, welches das zarte und dichte Netz zwischen den Zellen der normalen Neuroglia erzeugt, ist im Allgemeinen bei jenen Gliomen spärlich, wo die Zellelemente sehr in Ueberfluss sind, sodass ein ächtes Netz manchmal nicht dabei ist, sondern die Fäserchen rings um die Zellen wie Bündelchen, meist der Länge nach, liegen, und es scheint, dass sie mit den zelligen Elementen innerjich verbunden sind.

Zuweilen dagegen sind sie in unregelmässiger Weise zwischen. den Zellen vertheilt, und sie verlaufen, indem sie sich so gegenseitig durchflechten, dass - ein mehr oder weniger dünnes und dichtes Netz daraus entsteht, dessen Fäserchen entweder sich an die Zellen drängen oder durch sie hindurchgehen. -

Bei den einfachen Gliosen, von denen ich verschiedene, wirklich typische Fälle studiren konnte, sind die Fäserchen sehr zahlreich, von verschiedener Grösse und Länge, und erzeugen ein dichtes und dickes Netz, welches bei den mit der Weigert'schen Methode erhaltenen Präparaten sehr deutlich erscheint und an das Fibrin-Netz erinnert.

Die Zellen sind spärlich, immer viel weniger zahlreich, als bei den ächten Gliomen, und haben ausserdem weniger atypische Eigenschaften, id est, sie gleichen mehr den Zellen der normalen Neuroglia. -

Man bemerkt aber nicht wenige Ausnahmen, d. h. neben Typen, welche den Golgi'schen Astrocyten ähnlich sind, erscheinen dreieckige oder unregelmässige, manchmal verlängerte und spindelförmige, manchmal kugelförmige Zellen mit dicken und kurzen, schwach färbbaren Fortsätzen, welche von den aneinander gedrängten oder durch den Zellkörper gehenden Fäserchen scharf zu unterscheiden sind. 
Der Kern oder die Kerne sind gut erkennbar und nehmen bald den centralen Theil, bald die Peripherie des zelligen Körpers ein. Bei den Gliomen dagegen zeigen die zelligen Elemente immer die Charaktere einer grösseren und entschiedenen Atypie; sie nehmen den embryonalen Charakter einer übertriebenen Vermehrungs-Thätigkeit wieder an, aber nicht die morphologischen Eigenschaften, die sie bei den ersten Phasen der normalen Neuroglia-Entwicklung gewöhnlich haben, d. i. die Epithelzellen- und die Spongioblasten-Form. In der That treten viel grössere Zellen auf, bei denen der zellige Körper verlängert oder unregelmässig polygonal ist, mit dicken und kurzen Fortsätzen und manchmal mit Segmentirungen, welche der Zelle eine sehr seltsame Form geben. Der Kern oder die Kerne sind nicht immer gut unterscheidbar von dem protoplasmatischen Körper, welcher selbst sich blau bei den mit der Weigert'schen Methode behandelten Präparaten und roth bei den mit der Methode von van Gieson erhaltenen Präparaten färbt.

Diese meist zahlreichen Zellen sind manchmal sehr nahe an einander gerückt und vereinigen sich zuweilen, sowie diejenigen der Endotheliome, besonders bei den sich in der Tiefe der Hirnrinde entwickelnden Gliomen.

Nach dem bisher Dargestellten gründen sich die Unterschiede zwischen den ächten Gliomen und den Gliosen nicht nur auf grobe anatomische Eigenschaften, sondern auch auf histologische Einzelheiten.

Im Hinblick auf die biologischen Eigenschaften können wir sagen, dass man, während das Gliom alle Eigenschaften einer Geschwulst im engsten Sinne des Wortes hat, d. h. die der Entwicklungs-, der Bildungs- und der Wachsthums-Autonomie, während es mit einigen Störungen in der ersten Bildung der nervösen Centren verknüpft ist, die Gliosen als Hyperplasien oder wenig atypische Neubildungen bezeichnen kann, welche den Bindegewebs-Neubildungen in parenchymatösen Organen im Allgemeinen ähnlich sind, die sich nach und nach entwickeln, indem die Grund-Elemente des Parenchyms atrophisch werden oder sich zersetzen.

Es ist nicht leicht, die zwischen den Zellen der pathologischen Neuroglia und den normalen Neuroglia-Zellen vor- 
handenen Unterschiede zu bezeichnen, welche sich auf die Form, die Grösse, die Zahl der Kerne und das manchmal nicht unsichere Vorhandensein von protoplasmatischen Fortsätzen, die man immer von den Fäserchen leicht unterscheiden kann, beziehen.

Ich möchte auf Grund meiner Beobachtungen die Hypothese aufstellen, dass, je mehr diese Elemente sich dem embryonalen Typus nähern, desto grösser die Proliferations-Thätigkeit, und desto leichter der Verlust der normalen morphologischen Charaktere wird.

Als eine andere Embryonalitäts-Eigenschaft möchte ich die Spärlichkeit oder das Fehlen der Fäserchen um die Zellen der pathologischen Neuroglia hinzufügen. Das würde auch bei der normalen Entwicklung stattinden. In der That, bei meinen Markpräparaten von normalen Menschen-Embryonen von 3 bis 5 Monaten, die ich mit der Weigert-Methode, welche mir immer gute Ergebnisse gegeben hat, bereitete, ist es mir nicht gelungen, die Existenz von gefärbten Fäserchen um die Embryozellen zu boweisen, obgleich die nervöse Substanz frisch und ohne Leichen-Veränderungen war. -

Bei normalen Entwicklungszuständen würden die zur Neuroglia-Bildung bestimmten ektodermischen Zellen, wie sich aus meinen Präparaten ersehen lässt, eine Migrations-Thätigkeit haben, welche den einzelnen Elementen gestattet, die primitive Ebene zu verlassen und in das umliegende Gewebe einzudringen, wo sie sich durch Segmentirung oder directe Theilung vermehren, ferner einsinken, sich unförmlich verändern und zu Elementen werden, die man richtig Gliogene nennen könnte. Die Migrations-Thätigkeit ist mehr oder weniger tief mit der Bildungs-Thätigkeit dieser Elemente verknüpft, und die Migration kann sich nach allen Richtungen, aber am meisten excentrisch verwirklichen.

So können wir uns erklären, wie die Zellen der primitiven Medularplatte oder des primitiven Nervenrohres, welche nicht nervöse Elemente, sondern Stützzellen erzeugen, durch die die ganze Dicke der W:ände des genannten Nervenrohres bis zu der Peripherie des Markes oder der Gehirnbläschen, das ist unter den Hirnhäuten, wandern können, indem sie sich vermehren und in Zellen normaler Neuroglia verwandeln. Sobald nun 
diese sogenannten gliogenen Elemente den erwachsenen Zustand erreicht haben, stellen sie nicht mehr die Eigenschaften der Migrationszellen und auch nicht jene der Ependymzellen, aus welchen die Migrationszellen entstanden sind, sondern die eigentlichen Charaktere der Neurogliazellen vor.

Ob ein ähnliches Verfahren bei der Zellen-Entwicklung der pathologischen Glia stattfindet, ist sehwer zu sagen.

Es ist nicht ganz sicher, ob alle erwachsenen Neurogliazellen die embryonalen Eigenschaften wieder aufnehmen können, um neue Neuroglia zu bilden, oder ob die Aufgabe, die pathologische Neuroglia zu bilden, bloss einigen Gliazellen zufällt, welche ibre embryonalen Eigenschaften länger erhalten.

\section{III.}

Ueber die Anwesenheit von Epithel-Elementen innerhalb der Gliome.

Eine Frage, welche sich mit der Embryonal- oder Fötal-Entstebung gewisser Gliome verbindet und welche geeignet ist, die genetische Verknüpfung zwischen den ectoblastischen Elementen und der anomalen Neuroglia-Bildung zu beweisen, ist diejenige, welche sich auf das Vorhandensein von Epithelzellen im Inneren einiger Gliome bezieht. Diese Frage wurde von manchen Beobachtern erörtert, von denen ich Arnold ${ }^{1}$ ), Miura ${ }^{2}$ ), Hoffmann ${ }^{3}$ ), Buchholtz ${ }^{4}$ ) und Ströbe ${ }^{5}$ ) erwähne.

Die aus ihren Beobachtungen abgeleiteten Schlüsse stimmen in der Annahme überein, dass diese Epithel-Bildungen aus dem Epithel des primitiven Nerven-Canälchens oder aus dem die Gehirnbläschen bekleidenden entstehen. Das beweisen, sagt Stroebe, die Eigenschaften der Epithel-Elemente, insbesondere

1) A rnold, Gehirn, Rückenmark und Schädel eines Hemicephalus u. s. w., Ziegler's Beiträge Bd. XII, 1892.

2) Miura, Ueber Gliom des Rückenmarks und Syringomyelie, Ziegler's Beiträge Bd. XI, 1891.

3) Hoffmann, Zur Lehre von der Syringomyelie, Deutsche Zeitsehrift für Nervenheilkunde Bd. III, 1893.

9) Bnchholtz, Beitrag zur Kenntniss der Hirngliome, Archiv für Psychiatrie u. Nervenkranheiten Bd. XXII, 1891.

5) Stroebe, Ueber Entstehung und Bau der Gehirngliome, Ziegler's Beiträge Bd. XVIII H. III, 1895. 
die Eigenschaft, feine Wimperhaare und einen Fuss zu besitzen, der in feine Fasern ausläuft, die sich dem Gliafasernetz beimischen.

In dem von Stroebe ${ }^{1}$ ) beschriebenen Falle eines Glioms des linken Occipito-Parietallappens waren Erweichungsheerde an einigen Punkten der Geschwulst vorhanden, - von denen manche sehr klein $(40 \mu)$, manche wie ein Hirsekorn gross waren, innerhalb welcher Gliafasern und Zellen in körniger Zersetzung sich fanden. Neben diesen Erweichungsheerden bemerkte man Höhlen, deren Wände mit einer einfachen Epithelschicht von cubischen oder cylindrischen, regelmässig geformten Zellen bedeckt waren. Solche Höhlen fanden sich in der Zahl von 4 oder 5 in einer Geschwulstzone von schwammigem Aussehen und einem Durchmesser von etwa $4 \mathrm{~cm}$. Dieselben Höhlen erschienen manchmal so gross, wie der Durchschnitt des normalen Canals im Rückenmark. Eine von ihnen war viel grösser; sie erschien in einer Reihe auf einander folgender Schnitte bald längsoval, mit vorspringenden und eingesunkenen Umrisslinien, bald auch als ein längerer Schlauch mit winklig geknicktem Verlauf.

Die Epithel-Auskleidung dieser grossen, unregelmässigen Hohlräume zeigte sich bald einschichtig, bald mehrschichtig. Die Epithelzellen, welche die kleinsten Höhlen auskleideten, waren mit feinen Wimperhaaren versehen, welche in Präparaten nach Mallory tiefblau gefärbt erschieṇen und sich bis in das Zell-Protoplasma hinein gegen den Kern der Zelle hin verfolgen liessen. Die grösseren dieser mit Epithel ausgekleideten Hohlräume waren von einem mehr oder weniger vollständig herumreichenden Kranz von Zellen umgeben, welche in runden Häufchen oder länglichen, säulenförmigen Gruppen dicht bei einander lagen. Solche Zellen waren von verschiedener Form, bald cylindrisch, bald verlängert. Dieselben unterschieden sich in jedem Falle von den Geschwulstzellen durch das polymorphe Aussehen ihres Zellkörpers und durch das Vorhandensein von Pigmentkörnchen im Inneren derselben.

Der eben theilweise berichtete Fund dieses von Stroebe untersuchten Falles ist ohne Zweifel einer der wichtigsten und

1) Stroebe, a. a. O., Ziegler's Beiträge Bd. XVIII Heft III. 
am meisten beweisenden der in der Literatur der Gliome registrirten Fälle, weil er viel besser, als die von Miura, von Hoffmann und von Schultze beschriebenen Exemplare, geeignet ist, die Wichtigkeit dieser Epithel-Bildungen innerhalb des gliomatösen Gewebes darzustellen und die Gliom-Entstehung zu erklären. Er lässt uns vermuthen, dass die erste Entwicklung der gliomatösen Bildung ihren Ursprung in einer zelligen Proliferation hatte, welche ihren Ausgangspunkt von dem Epithel der anomalen, schlauchförmigen Sprossen des primitiven Neuralrohres genommen hat.

Nach Stroebe wäre dann der von Protoplasma-reicheren, in Gruppen vereinigten und einige der Zellschläuche umgebenden Zellen gebildete Wall als ein späterer Nachwuchs der Cylinderepithelzellen aufzufassen, während die Elemente des gliomatösen Gewebes Abkömmlinge der primitiven Epithelien sein würden, welche das ursprüngliche Wachsthums-Centrum des gliomatösen Gewebes darstellen würden.

Andererseits würden die neuen gliomatösen, von den Mutterzellen abgeleiteten Zellen eine eigene und unabhängige Thätigkeit besitzen, sich durch Theilung mehr oder weniger langsam, je nach der kleineren oder grösseren Wachsthums-Schnelligkeit des Glioms, vermehren. Auf solche Weise wäre es möglich, das marginale, infiltrative Wachsthum des Glioms zu erklären.

Wie man bemerken kann, meint Stroebe, dass die Zellen der gliomatösen Neubildung nicht von denjenigen Epithel-Elementen unmittelbar entstehen, welche, in kleinen Gruppen vereinigt, einen Kranz um die primitiven, von cylindrischem Epithel bedeckten Sprossen bilden, sondern er glaubt, dass dieselben von diesen letzteren direct abstammen; somit würden die runden, platten, um die Röhrchen herum liegenden Zellen keine Uebergangs-Elemente bilden.

Epithel-Bildungen hat nun auch Miura ${ }^{1}$ ) beobachtet in einem Falle von Rückenmarks-Gliom mit Bildung einer Höhle, deren Wände mit einer Schicht von Epithelzellen in einer gewissen Strecke ganz bedeckt waren; diese Zellen glichen denjenigen, welche den mit der neugebildeten Höhle communicirenden Canal bedeckten. Die Höhle war durch eine partielle

1) Miura, a. a. 0. 
Zerstörung und Resorption des gliomatösen Gewebes erzeugt worden, und das sich vermehrende Epithel des Centralcanales war in diese Höhle eingedrungen, indem es einen Theil der vorderen Wand derselben bedeckte.

Obgleich dieser Fall, wie andere, die Möglichkeit bestätigt, dass gewisse secundäre, mit dem Centralcanal communicirende oder nicht communicirende Höhlen (Syringomyelie) sich in dem gliomatösen Rückenmark bilden können, so giebt er doch keinen Beweis für die Entstehungs-Verbältnisse des Neuroglia-Gewebes, welches von den Epithelien innerhalb der erwähnten Höhlen neu gebildet wurde.

Die Ansicht Miura's über die Entstehung der Syringomyelie in dem von Gliomen eingenommenen Rückenmark theilt auch Schultze ${ }^{1}$ ). Hoffmann ${ }^{2}$ ) dagegen, der bei seiner interessanten Arbeit über die Syringomyelie ein reiches Beobachtungs-Material benutzte, hat die Meinung ausgesprochen, dass die EntstehungsUrsache der Syringomyelie in der Mehrzahl der Fälle in angeborenen Anomalien liege, die sich durch die Anwesenheit von Keimgewebs-Resten, d. h. von Sprossen embryonaler Elemente, meist hinter dem Centralcanal längs der Verschlusslinie, äussern. Solche Reste des primitiven Nervenrohrs könnten in einer späteren Lebenszeit der Ausgangspunkt einer Gliom-Bildung-werden, welche in mehr oder weniger ausgedehnten Strecken das Mark durchsetzen und in ihrem Inneren nekrobiotische Erweichungs- und Zerstörungszonen zeigen würde, aus denen dann secundäre, die anatomische Grundlage der Syringomyelie bildende Nebenhöhlen entstehen könnten.

Anch Hoffmann nimmt an, dass die innerhalb der Gliome gefundenen Höhlen oder Räume in secundärer Weise durch partielle Zerstörung und weitere Resorption des gliomatösen Gewebes zu entstehen pflegen.

Er ist aber nicht der Meinung von Schultze über die Entstehung des Epithels innerhalb der obengenannten Höhlen, denn er leugnet, dass dieses Epithel selbst eine secundäre, in die durch

1) Sebultze, Fr., Klinisehes und Anatomisches äber Syringomyelie,

Zeitschr. f. klin. Medicin Bd. XXIII, 1888.

2) Hoffmann, a. a. 0 . 
der Zersetzung des gliomatösen Gewebes entstehenden Höhlen eindringende Bildung darstelle.

Die Vorstellung, dass man die anomale und heterotopische Epithel-Bildung für unabhängig und primitiv, sei es in Bezug auf die Entstehung der Neuroglia, sei es in Bezug auf das Erscheinen von Höhlen (Syringomyelie) halten kann, wird durch die oben erwähnte Stroebe'sche Beobachtung und ferner durch die andere von Kahler und $\mathrm{Pick}^{1}$ ) bestätigt, welche sich auf einen Syringomyelie-Fall bezieht, der vielleicht von einem durch abnormen Verschluss des centralen Canals entstandènen Divertikel ausgegangen ist. -

Es scheint darnach ganz sicher zu sein, dass von Verlagerungen des primitiven Epithels des Centralcanales laterale Sprossungen sich herleiten können, welche sich gewöhnlich im Inneren der Räume oder Höhlen finden, um welche herum Neuroglia-Gewebe sich neu bildet und verdichtet.

Das Verhältniss zwischen dem Erscheinen dieser Epithelien und der neuen pathologischen Neuroglia-Bildung ist noch nicht genügend erklärt worden; ebensowenig kennen wir die EntstehungsBedingungen der neuen pathologischen Neuroglia im Verhältniss zu der Anordnung des anomalen Epithels, d. h. es ist nicht immer leicht zu bestimmen, ob wir es mit einem angeborenen Falle, mit einer Entwicklungs-Störung, oder mit einer pathologisch erworbenen Erscheinung zu thun haben. Mit der Absicht, diese letzten Fragen insbesondere zu lösen, habe ich mich mit dem Studium eines von mir im linken Gehirnlappen eines 6 jährigen Kindes gefundenen Falles von Gliom beschäftigt, welches besonders den Gyrus uncinatus und einen beträchtlichen Theil der unterliegenden weissen Substanz einnahm.

Bei diesem in der Höhle des linken Seiten-Ventrikels entstandenen und von derselben mittelst einer weissen, ganz normalen Substanz getrennten Gliom habe ich manche, unregelmässig in den verschiedenen Geschwulsttheilen zerstrente Epithelial-Bildungen gefunden, welche bald als Auskleidungen von engen Spalten, bald als kleine, innerhalb des verdünnten Neuroglia-Gewebes zerstreute Inseln oder Gruppen, aber immer

1) Kahler und Pick, Prager Vierteljahrsschrift Bd. 14 S. 142. 
ohne jede Verbindung mit Divertikeln der centralen Höhlen, erschienen.

Solche Epithel-Bildungen waren auch bezüglich ihrer morphologischen Eigenschaften sehr interessant, da sie sich von den normalen Epithelien und von den bei gewissen Gliomen beschriebenen Epithelien durch den Mangel an Wimperhaaren und durch die manchmal unregelmässige Zellform und auch durch ihre Verhältnisse zu den Neuroglia-Fasern und Zellen sehr unterschieden.

In diesem von mir untersuchten Falle habe ich eine dünne, nicht mit blossem Auge, sondern nur im Durchschnitte und bei einer Vergrösserung von 20-40 im Durchmesser sichtbare Spalte beobachtet, welche Anfangs so enge war, dass die Ränder fast in Berührung standen, die sich dann aber in der Tiefe weiter ausdehnte oder enger wurde, je nachdem die Geschwulst ihre Dicke oder die Richtung wechselte.

Eine solche Spalte zog durch die ziemlich glatte Oberfläche der den ganzen linken Gyrus uncinatus und theilweise auch die benachbarten Windungen betreffenden Geschwulst; sie erschien von einem einschichtigen Epithel bekleidet, dessen Zellen cylindrisch oder cubisch oder oval und mit gut differenzirten Kernen versehen waren (Taf. XIII Fig. 1-2).

An einigen Punkten wurde diese Epithel-Auskleidung durch Entartung und Trennung der zelligen Elemente unterbrochen, an anderen waren die Zellen zahlreicher und so vereinigt, dass sie konische oder papilläre Erhebungen bildeten, welche nach dem Centrum der Spalte hin vorragten und manchmal fast den ganzen Raum derselben einnahmen.

Die einzelnen Zellen sehen nun, wie es an der genau reproducirten Figur meiner histologischen Präparate deutlich zu Tage tritt, wie Epithelzellen aus.

Bei starker Vergrösserung zeigen sie ein homogenes Protoplasma, welches farblos bleibt, während es sich bei den WeigertPräparaten leicht gelblich fürbt. Aus dem Zellkörper treten weder Fortsätze, noch Wimperbaare hervor, und die Zellen sind meistens cubisch, manchmal jedoch oval und unregelmässig.

Diese letzteren Formen finden sich insbesondere an den freien Zellen innerhalb des Spaltraumes oder in den mehr ober- 
flächlichen Zellen jener Gruppen, welche wie papilläre Erhebungen angeordnet sind.

Auf einigen Strecken des Epithel-Gewebes der Spalte sind die Zellen mehr platt, und ihre Körper einander so nahe gerückt, dass es nicht möglich ist, die laterale Grenze zu unterscheiden, und dass das Protoplasma einer Zelle sich in das der benachbarten Zellen fortsetzt; man bemerkt also eine Art von protoplasmatischem, mit Kernen versehenem Band, welches an der Grenze des Raumes oder der Spalte steht.

Die Kerne dieser Zellen sind im Allgemeinen gut differenzirt, denn sie färben sich nach der Weigert'schen Methode stark blau, während das Protoplasma eine gelbliche Nuance annimmt.

Unmittelbar unter dieser Epithel-Auskleidung, die wahrscheinlich einen Abkömmling des primitiven Neuro-Epithels darstellt, beobachtet man ein dichtes Netz von Neuroglia-Fäserchen, die sich in verschiedener Art durchflechten und sich durch ihre schwarzblaue Tinte in den nach der Weigert'schen Methode gefärbten Präparaten scharf differenziren. Einige dieser Fäserchen dringen in die Epithelzellen ein, und zwar genau dort, wo die in kleinen Gruppen vereinigten Zellen sich nicht in unmittelbarer Berührung finden. Andere Fäserchen treten an das Lumen der Spalte heran, gerade da, wo das Epithel nicht continuirlich ist, und bilden eine Art von Schopf, in dessen Mitte manchmal Epithelzellen vorhanden sind. Man kann nicht sicher erkennen, ob diese Fäserchen von dem Körper der oben genannten Zellen ausgehen, mit denen sie übrigens keine sehr intime Beziehungen haben. -

Aber ausser diesen, regelmässig in der Art einer Auskleidungsschicht auf die Wände der oben erwähnten Spalte vertheilten Epithelien, welche an die von Ströbe bei seinem Falle beschriebenen und als Reste des primitiven nervösen Canals betrachteten Epithel-Bildungen erinnern, habe ich in dem von mir untersuchten Falle eines Glioms des Schläfenlappens und in einem anderen Vierhügel-Gliom, Gelegenheit gehabt, die Anwesenheit von Epithelzellen-Gruppen innerhalb des Gliomgewebes zwischen den Maschen des Fasernetzes zu beobachten, ohne dass wirkliche Räume oder Spalten um dieselben herum 
vorhanden waren (Taf. XIII Fig. 2 cc). An einigen Punkten habe ich auch manche, in dem Fasernetze isolirte Epithelzellen getroffen; zwischen dem Zellkörper und den näheren Fäserchen fand sich nur manchmal eine leichte Verdünnung dieser Fäserchen.

Diese Zellen können von den nervösen Zellen durch ihre Eigenschaften unterschieden werden, nehmlich durch ihre cubische und eiförmige Gestalt, durch das Fehlen von protoplasmatischen Fortsätzen und grossen Kernkörperchen, und durch die verhältnissmässig kleinen Kerne. Dieselben lassen sich von den noch in der Entwicklungs-Periode befindlichen NeurogliaZellen durch die scharfe Differenzirung des im Verhältniss zu der Menge des Protoplasmas sehr kleinen Kernes und durch die grössere Regelmässigkeit des zelligen Körpers, welcher sich bei den W eigert-Präparaten leicht gelblich gefärbt zeigt, unterscheiden. Sie sind endlich von den Leukocyten verschieden.

Es ist mir niemals gelungen, irgend eine karyokinetische Figur an den Kernen dieser Zellen zu finden, was vermuthen lässt, dass dieselben sich durch directe Theilung vermehren, während sie den embryonalen Charakter bewahren.

Die Thatsache, dass diese Elemente von epithelialem Aussehen nicht nur in ihrer besonderen Anordnung die Wände gewisser cystischer Räume oder Spalten bedecken, welche manchmal in der Tiefe der Gliome vorhanden sind, sondern dass sie auch in kleinen Gruppen oder isolirt innerhalb des GliomfäserchenNetzes verstreut sind, lässt ersehen, dass diese Elemente sich zu derselben Zeit, wie die Glia-Elemente, vermehren, und dass dieRäume oder Spalten der Epithelzellen-Bildung nicht immer vorher bestehen, sondern manchmal ganz und gar fehlen, manchmal in secundärer Weise nach der Entartung und dem Verschwinden des Gliomgewebes entstehen.

Solchem Verschwinden würde eine neue Vermehrung der Epithelzellen folgen, welche sich in Reihen neben einander anordnen, um eine Bekleidung der Wände der neuen Höhlen zu bilden, oder Gruppen und Schnüre von unregelmässiger Form: zu erzeugen.

Man kann also annehmen, dass diese Epithel-Elemente, die sich in der Tiefe des Glioms finden, eine zweifache EntstehungsMöglichkeit besitzen, obwohl man sie als Abkömmlinge des Epithels- 
des primitiven nervösen Canales betrachten kann. : So viel auch die wenigen bis jetzt beschriebenen Fälle uns zu urtheilen gestatten, können wir die Anwesenheit von Epithel-Bildungen im Innern der Räume oder der cystischen Höhlen, die sich in der Tiefe einiger in den centralen Theilen der cerebro-spinalen Achse entstehenden Gliome finden, mit der Annahme erklären; dass solche Räume nichts anderes, als Divertikel oder Abzweigungen der Centralhöhlen seien, und dass das sie bekleidende Epithel eine Fortsetzung des Epithels des primitiven nervösen Canals sei, so dass die Gliom-Bildung in diesen Fällen an einen Entwioklungsfebler der nervösen Centra anknüpfe.

Mit dieser Annahme ist es möglich die Entstehung mancher mit Syringomyelie verbundenen Markgliome (Mi u ra) und mancher an den Wänden der lateralen Ventrikel (Hartdegen) sich entwickeloden Gliome zu erklären.

Da aber in der Mehrzahl der Gliomfälle eine Verbindung der Neoplasma-Entwicklung mit dem Entwicklungsfehler des entsprechenden Theiles des Central-Nervensystemes, d. h. mit Divertikeln der Centralhöhlen, nicht vorhanden ist, so ist anzunehmen, dass die Verlagerung der Epithelien auch in einer anderen Weise stattfinden könne, und dass der Einschluss derselben im Innern des Gliom-Gewebes eine active Thätigkeit, d. h. eine wirkliche Migration darstelle. Für jene Gliome, die in der Nähe der Centralhöhlen wachsen, darf man wohl die Möglichkeit annehmen, dass die Geschwulst, indem sie wächst und regressiven Metamorphosen anterworfen wird, in die Wände der Centralhöhlen eindringen und durch Spalten oder Räume mit diesen centralen Höhlen in Verbindung treten kann. Die sich durch diese Verbindungswege vermehrenden Auskleidungs-Epithelien würden sich in die eben genannten Räume einschleichen und die Wände derselben mehr oder weniger vollständig bedecken.

Auch in diesen Fällen wäre es nicht leicht, die secundären Epithel-Bildungen von epithelialen, mit Entwicklungsfehlern der nervösen Centren verbundenen Neubildungen zu unterscheiden.

Die Annahme der Migrations-Möglichkeit der NeuroepithelElemente darf man aber nicht ron der Hand weisen; sie findet ihre rationelle Begrüudung in dem Verhalten der Epithelzellen 
während der normalen Entwicklung und ferner in dem histólogischen Befund gewisser oberflächlicher Gliome, bei welchen man, wie bei dem von mir beschriebenen Falle, epitheliale, ganz von den inneren Centralhöhlen-Auskleidungen unabhängige Bildungen fand. Der Befund von epithelförmigen Elementen innerhalb des Gewebes von Gliomen, die sich in verschiedenen, von den Centralhöhlen weit entfernt liegenden Gehirnzonen (Windungen) entwickelt hatten, ohne dass eine Communication mit denselben oder irgend eine Beziehung zu den ventriculären Höhlen zu finden war, führt uns zu der Annahme, dass es sich um Zellen des Neuroepithels handelt, welche nicht längs der vorher bestehenden Divertikel des primitiven nervösen Canals, sondern durch die, um die genannten Hohlräume herum liegende nervöse Substanz ausgewandert sind.

Diese Migration findet sehr wahrscheinlich während der Entwicklung der Nervencentren statt, und es ist möglich, dass die Epithel-Elemente sich in diesem heterotopischen Zustand lange erhalten, als Embryonal-Elemente, von denen das Gliom später erzeugt werden könnte.

Demnach wäre angeboren nur die Heterotopie des primitiven Neuroepithels in Form kleiner Gruppen oder Zellenschnüre; die Gliom-Entwicklung erfolgt wahrscheinlich lange Zeit nach der Geburt.

Nach dieser Erklärung des Vorhandenseins von EpithelzellenInseln oder -Schnüren im Innern des gliomatösen Gewebes könnte man nicht nur eine angeborene Disposition im Sinne Cohnheim's bei gewissen Gliomen annehmen, sondern auch die Entwicklung der pathologischen Neuroglia der der normalen gleichstellen.

Es ist in der That bekannt, dass die Ganglienzellen und die nervösen Fasern (Neuroblasten) ebenso, wie die Neurogliazellen (Spongioblasten), von dem Epithel des primitiven nervösen Canals abstammen, und dass die letzteren nichtin ihrem Erzeugungsgebiet bleiben, sondern, indem sie sich vermehren, die Oberfläche des nervösen Canals verlassen, um ihre bleibenden Stellungen im Gehirn und Rückenmark einzunehmen. Darf man nun an eine Analogie zwischen dem normalen und dem pathologischen Entwicklungs-Process denken? Eine solche Analogie 
würde sich nicht nur auf die Entstehung der Elemente der pathologischen Neuroglia von dem Neuroepithel oder von seinen $A b$. kömmlingen, sondern auch auf die Migrations-Möglichkeit der sich neubildenden Neurogliazellen beziehen. Jedenfalls würden sich tiefe. Unterschiede zwischen dem normalen und dem pathologischen Entwicklungs-Process zeigen, aber man könnte sie mit der Annahme erklären, dass nicht normales Nervengewebe, sondern nur pathologisches Gewebe aus heterotopischem Epithel und unter anormalen Bedingungen gebildet werden kann. Und da dieses pathologische Gewebe fast ausschliesslich aus Neuroglia besteht, muss man zu dem Schlusse kommen, dass bei den verlagerten Epithelien die Elemente fehlen, welche Neuroblasten bilden können, während sich in grosser Zahl diejenigen finden, welche fähig sind, Spongioblasten zu bilden, obwohl es nicht ausgeschlossen ist, dass viele von diesen pathologischen EpithelElementen die Differenzirungs-Möglichkeit verloren haben und deshalb weder Nervenzellen, noch Neurogliazellen erzeugen, sondern als unthätige Elemente im Innern der gliomatösen Neủbildung bleiben.

Aus Allem, was ich bis jetzt vorgetragen habe, würde folgender Schluss hervorgehen:

Die in der Tiefe der Gliome befindlichen, manchmal wie einfache Auskleidungs-Schichtender cystischen Hohlräume ausgebreiteten, manchmal in kleinen Gruppen vereinigten oder aus dem primitiven Nervencanal heraustretenden, Zellenschnüre bildenden Epithelzellen stellen nicht immer Neuroepithel-Sprossen dar, welche durch Fehler erster Bildung verlagert oder einfach in der Tiefe der gliomatösen Neubildung eingeschlossen und mit keiner Bildungsthätigkeit ausgestattet sind; sondern sie zeigen sich als ektodermische Zellen, welche den embryonalen Typus und die Eigenthümlichkeit bewahren, durch das normale, neryöse Gewebezu wandern, und welche, während sie nichtgeeignet sind, sich in Neuroblasten zu differenziren, um Ganglienzellen und nervöse Fäserchen bildenzu können, ejnen der wichtigsten Entwicklungsplätze für gliomatöse Neubildung darstellen. 
Dass die Zellen der pathologischen Neuroglia direct von dem Epithel sich ableiten, welches die Centralhöhlen auskleidet, ist durch unmittelbare Wahrnehmung zu beweisen unmöglich, während man das für die normale Neuroglia beweisen kann. Jedenfalls scheint es der Hypothese von Ströbe günstig zu sein, dass sie nur die erste Entwicklung der gliomatösen Neubildung von Epithelkeimen ableiten will, welche aus dem primitiven nervösen Canal ausgewandert sind, während die zelligen Elemente, welche die folgende glimatöse Neubildung charakterisiren, die Fähigkeit haben müssten, sich in unabhängiger Weise im Verhältniss $z u$ der grösseren oder geringeren Gliom-Thätigkeit za vermehren.

Diese Ansichtsweise gewinnt an Wahrscheinlichkeit, indem sie sich auf die Betrachtung stätzt, dass eine Omwandlung von Epithelzellen in Neurogliazellen nicht bewiesen ist, und indem sie auf das Vorhandensein von gewissen, grossen, regelmässig rundlichen, Protoplasma-reichen, mit einigen dicken und kurzen Fortsätzen versehenen Zellen hindentet, deren Kern klein und gut differenzirt ist. Die Anwesenheit dieser Zellen habe ich in manchen von mir untersuchten Gliomen bestätigt; dieselben könnten, meiner Meinung nach, den Namen vong gliogenetischen Zellen erhalten.

Bei dieser histologischen Besonderheit möchte ich mich noch etwas aufhalten.

Es handelt sich um zellige Elemente, welche sich meistens. isolirt inmitten eines dichten Fäserchennetzes zeigen. Einige dieser Fäserchen drängen sich so dicht an den zelligen Körper, dass sie eine Emanation desselben zu sein scheinen; andere gehen durch den Körper selbst, so dass sie als Fortsätze erscheinen.

Diese Zellen sind platt, bald oval, bald von unregelmässiger Form. Ihr Protoplasma färbt sich in den nach der Weigertschen Methode behandelten Präparaten leicht blau; die Färbung wird jedoch intensiver gegen die peripherischen Theile hin, wo sich die Neuroglia-Fäserchen herandrängen. Der Kern erscheint manchmal gut erkennbar, klein, rundlich oder verlängert, manchmal ist derselbe am Centrum, andermal an der Peripherie gelegen (Taf. XIII Fig. 4, 5). Manchmal wird er von Fäserchen verdeckt, welche durch die Zellen verlaufen. 
Neben diesen grossen, platten Zellen sieht man bei einigen Gliomen kleinere, ovale Zellen, in welchen der Kern vom Protoplasma nicht gut differenzirt ist; das letztere erscheint dann, in den nach der $W$ eiger t'schen Methode behandelten Präparaten, leicht bläulich. Solche kleineren Elemente sind Protoplasma-Klümpchen ähnlich und kommen an den nodulären Punkten mancher Fäserchen zuweilen vor. Diese Zellen sind zahlreicher in jenen Gliomen, wo Epithel-Bildungen sich finden; sie bilden meiner Meinung nach ein Zwischenstadium $z$ wischen Epithel- und Neurogliazellen: sie nähern sich durch einige Charaktere, durch ihr Volumen, durch ihre abgeplattete Form, durch ihren Mangel an ächten Fortsätzen und durch die Differenzirung des Kerns, ferner durch die Art ihrer Vereinigung, wo sie zahlreich sind, mehr den Epithel- oder Endothelzellen. Durch andere Eigenschaften dagegen, wie durch ihr intimes Verhältniss zu den Fäserchen der neugebildeten Neuroglia, nähern sie sich mehr den Stützzellen. Man darf aber nicht vergessen, dass wir von pathologischer Neuroglia sprechen. Eine für mich sehr interessante histologische Besonderheit, welche diese Zellen von den Epithelzellen unterscheidet, bezieht sich auf die Art ihrer Vermehrung, die ich an zwei verschiedenen Gliomen studiren konnte: der eine Fall betraf ein 15 jähriges Mädchen (Lazzaro), der andere einen 35 jährigen Mann (Agostini).

In manchen nach $W$ eigert behandelten Präparaten von solchen Gliomen habe ich 4 bis 12 Gruppen von ovalen Kernen innerhalb des Netzes von Neuroglia-Fäserchen gefunden (Taf. XIV Fig. 1, 2). Diese Kerne besassen wenige Chromatinkörnchen und waren stark blau gefärbt. Sie sind dicht an einander gedrängt, wie man in der getreu wiedergegebenen Abbildung des Präparates sehen kann; sie bilden eine unregelmässige Gruppe, als ob sie aus der Theilung eines sprossenden Keimes entstanden wären, aber sie sind in Reihen angeordnet, wie die Kerne der Maskelzellen wäbrend des Regenerations-Processes der gestreiften Fasern (Taf. XIV Fig. 1).

Offenbar beweist dieser Befund, dass es sich um Zellen handelt, welche sich durch directe Theilung des Kernes vermebren, und dass von jedem dieser neugebildeten Kerne eine neue Neurogliazelle entstehen kann. Sehr selten sieht man 
Neuroglia-Fäserchen, welche sich zwischen die sich vermehrenden Kerne einschieben; meistens ist die durch directe Theilung gebildete Kerngruppe in einem von dem Netze umgebenen Raum gelegen, was beweist, dass jede solche Kerugruppe von einer einzigen Zelle abstammt.

Ich habe oben erwähnt, dass diese von mir „gliogen“ genannten Zellen Abkömmlinge der im Innern einiger Gliome liegenden Epithelzellen darstellen könnten. Da ich aber dieselben auch bei manchen anderen, und zwar oberfächlichen (dicht an der Gehirnrinde befindlichen) Gliomen gefunden habe, bei welchen ich keine ächten Epithel-Bildungen bemerkte, so ist es denkbar, dass diese Zellen in verschiedener Weise erzeugt werden können, und dass sie einen embryonalen Typus von Neurogliazellen der pathologischen Neubildungen darstellen. Wenn man auch absolut keine Reste von Epithel des Nervenrohres in gewissen Gliomen findet, so ist es doch nicht auszuschliessen, dass solche Reste entartet oder verschwunden seien. Auf diese Art könnte man die Gliome erklären, welche sich bei Erwachsenen weit von den Centralhöhlen, wo die Epithelien sich finden, entwickeln (Gliome der Gehirnrinde, des Balkens, der Hirnganglien).

Die oben beschriebenen, Epithel-förmig aussehenden, von mir in manchen sehr schönen Gliom-Exemplaren gefundenen und als gliogenetische. bezeichneten Zellen erinnern an die von Buchholtz ${ }^{1}$ ) 1891 beschriebenen aus einem Gliom des Frontallappens einer 40 jährigen Frau. In jenem Falle aber fanden sich diese Elemente so reichlich und so dicht an gewissen Geschwulstpunkten, dass sie das Aussehen von Epithelzellen in verschiedener Weise nahmen. Die Bedeutung dieser zelligen Elemente hat Buchholtz nicht mit Sicherheit dargelegt. Obwohl er wegen ihrer Aehnlichkeit mit den Auskleidungszellen der Oberfläche der Gehirnventrikel und des Centralmark-Canals annehmen konnte, dass er es mit einer Neubildung von Ependymzellen zu thun hatte, welche zur Entstehung einer Art von Divertikel der Seitenventrikel-Höhle geführt hatte, hat el sich gegen diese Hypothese ausgesprochen, weil der Ort, wo diese

3) Buchholtz, Beitrag zur Kenntniss der Hirngliome. Arch. f. Psych. u. Nervenkrankh. Bd. 22 , S. 385 . 
Zellen-Anhäufungen gefunden wurden, weit von der VentrikelOberfläche entfernt lag, und weil es ihm trotz vieler, in Reihen ausgeführter Schnitte nícht gelang, eine Verbindung dieser zelligen Schichten mit der Ventrikel-Oberfläche festzustellen. $\mathrm{Da}$ er nun erkannte, dass die Stützzellen der nervösen Elemente nichts anderes, als Abkömmlinge des äusseren Keimblattes sind, schien es ihm nicht unmöglich, dass die Neurogliazellen während ihrer pathologischen Neubildung eine solche Form wieder annehmen könnten, durch die sie den Epithelzellen gleich werden, von denen sie abstammen.

Diese von Buchholtz nur vermuthete Thatsache wird durch meine Präparate, namentlich durch die nach Weigert gefärbten, deutlich beweisen. Die Zellen in diesen Präparaten kann man leicht von den Nervenzellen unterscheiden, welche ungefärbt $\mathrm{zu}$ bleiben pflegen und die bekannten protoplasmatischen Fortsätze besitzen. Bei den an der Gehirn-Oberfläche liegenden Gliomen (Taf. XIV Fig. 5, Fälle Baessato und Rasi) zeigen sie sich so zahlreich und in solcher Art vereinigt,-dass sie an gewisse Endotheliome erinnern. Sie sind in der That gross, platt, bald rundlich, bald länglich, bald dreieckig, bald unregelmässig; der Kern ist ziemlich gut differenzirt, meistens einfach, klein, central, selten zweifach. Das Protoplasma ist homogen, reichlich und färbt sich schwach schmutzigblau, fast aschgrau an den nach der Weigert'schen Methode behandelten Präparaten. Einige Theile der Oberfläche dieser Zellen zeigen sich stärker blau gefärbt, und es scheint, dass zarte Fäserchen aus diesen Theilen entstehen, welche, indem sie sich nach der Richtung des grösseren Durchmessers der Zelle selbst verbreiten, von Zelle zu Zelle gehen und inmitten des umgebenden verwickelten Netzes verschwinden. Bei einigen der von mir untersuchten Rinden-Gliome waren diese Zellen an der Oberfläche weniger zahlreich, namentlich dort, wo die Fäserchen überwogen (Molecular-Schicht), während sie in den tieferen, den verschiedenen Ganglienzellen-Schichten und der weissen Subcortical-Substanz entsprechenden Theilen immer zahlreicher wurden und immer von Fäserchen umgeben waren. Manchmal waren diese Zellen in grosser Zahl aneinander gedrängt, so dass sie Schnüre von cylindrischer oder unregelmässiger Form bildeten, 
um welche herum ein dichteres Netz von Fäserchen vorhanden war, innerhalb. dessen man neugebildete Blutgefässe sah. Wegen dieser Anordnung erinnerten diese Geschwulsttheile an die Endotheliom-Structur. Eine solche Aehnlichkeit muss uns aber die ectodermische Entstehung der Gliazellen nicht vergessen lassen; sie kann andeuten, dass die Gliazellen in pathologischen Zuständen wegen ihrer schnell auf einander folgenden Vermehrung morphologische Eigenschaften angenommen haben, durch welche sie den EctodermaZellen, von denen sie berstammen, gleich erscheinen.

Diese Aehnlichkeit ist aber. sehr relativ, sie kann also eine Verwechslung mit ectodermalen Zellen nicht ermöglichen. In einigen Zonen dieser Gliome waren die Zellen rundlich und in gewissen Räumen gelegen, welche von Fäserchen oder von sich durchflechtenden Fäserbündeln begrenzt waren, so dass sie sich bezüglich der das Stroma bildenden bindegewebigen Fäserchen wie die Elemente gewisser Krebse verhielten. Wahrscheinlich sind die grossen, als neugebildete Ganglienzellen bei einigen Gliomen beschriebenen Zellen nichts anderes, als Neurogliazellen von embryonalem Typus. Damit darf man aber nicht ausschliessen, dass in sehr seltenen Fällen ächte ganglionäre Zellen sich innerhalb des Neuroglia-Gewebes finden; bei den erworbenen Gliomen aber sind solche Zellen gewöhnlich nicht neugebildet, sondern sie stellen die schon vorher vorhandenen nervösen Zellen dar, welche mehr oder weniger von der neuen Neuroglia modificirt worden sind.

Nach Ströbe ist die Anwesenheit von übergebliebenen nervösen Fasern und von ganglionären Zellen bei Gliomen ein nicht sehr ungewöhnlicher Befund. Der Verfasser aber spricht sich nicht über die Entstehung und die Natur der erwähnten Zellen aus, sondern or ist nur überzeugt, dass dieser Befund dazu dient, die gemeinsame ectodermische Enstehung der Neuroglia und der ganglionären Zellen zu erklären.

\section{IV.}

Verschiedene Zellenformen der pathologischen Neuroglia.

Aus alle dem, was ich oben auf Grund meiner eigenen Beobachtungen über die Structur der Neurogliazellen bei der 
pathologischen Neuroglia-Bildung gesagt habe, geht hervor, dass diese Zellen manchmal gar nicht den Deiters'schen Zellen gleichen.

Diese Veränderungen stehen in Zusammenhang mit der Aufhebung ihrer physiologischen Function, als Stützzellen für die nervösen Elemente (Ganglienzellen und Nervenfasern) zu dienen, und mit der Wiederaufnahme einiger embryonaler Eigenschaften, wie diejenigen der Vermehrungs- und der Migrations-Thätigkeit.

Obwohl die morphologischen Charaktere sich während der pathologischen Neubildung ändern, nehmen die Neurogliazellen nicht immer den Typus der bindegewebigen Elemente an, sondern einige von ihnen besitzen besondere Eigenschaften, derenwegen sie an ectodermale Elemente, aus denen sie entstehen, erinnern.

Eine Eigenschaft, durch welche die Neurogliazellen sich mehr den bindegewebigen Elementen nähern, besteht nicht nur in der Form des Zelleibes, sondern auch in ihren Verhältnissen zu den Fäserchen, Verhältnisse, die bei pathologischen Zuständen intimer, als bei den normalen, sein werden.

Wenn man nun die in den pathologischen NeurogliaBildungen befindlichen Zellen von einem rein morphologischen Standpunkte studiren will, so kann man folgende verschiedene Formen darstellen.

1. Kleine runde, $(7-10 \mu)$ mit rundem, stark färbbarem, von einem dünnen, hyalinen, durchsichtigen protoplasmatischen Mantel umgebenem Kerne versehene Zellen. Nur bei der Untersuchung von Dilacerations-Präparaten aus frischen Gliomstückchen mit Immersionslinse ist es möglich, diesen unfürbbaren Protoplasma-Mantel zu sehen. Bei den gefärbten Schnitten sieht man den Kern nur im Innern eines Neuroglia-Fasernetzes. Solche Zellen finden sich vorzüglich in den peripherischen GliomTheilen und an den einfachen Gliosen, sie sind denjenigen der normalen jungen Neuroglia gleich und entsprechen den Deitersschen Zellen.

Diese Zellen scheinen bei den nach der Mallory'schen und Weigert'schen Methode behandelten Präparaten nicht mit protoplasmatischen Fortsätzen versehen zu sein; sie werden aber manchmal von ausserordentlich feinen Neuroglia-Fäserchen durchbrochen. 
2. Grössere, längliche, ovale oder spindelförmige Zellen; mit einem $10-15 \mu$ langen, stark färbbaren, zuweilen ChromatinKörnchen enthaltenden, von geringem Protoplasma umgebenen Kerne. Dieses Protoplasma ist an den peripherischen Theilen, an welchen die Neuroglia-Fäserchen nicht selten sehr dicht gedrängt stehen, schwach färbbar. Die Fäserchen verlaufen mehr in der longitudinalen Richtung des zelligen Elementes und verhalten sich wie bindegewebige Fäserchen den fixen Zellen desselben Bindegewebes gegenüber. Solche Fäserchen gehen über das polare Ende der Zelle hinaus und bilden gewissermaassen einen Fortsatz der Zelle selbst, welche alsdann mit zwei dünnen, polaren Fortsätzen versehen erscheint. Diese Zellen finden sich insbesondere bei jenen Gliomen oder jenen Gliom-Theilen, in welchen parallel angeordnete Bündel von Fäserchen im Ueberfluss vorhanden sind.

3. Grosse, dreieckige oder unregelmässig polygonale Zellen, von deren Ecken grosse, dicke, bald kurze, bald längere Fortsätze sich trennen, welche nur selten Verzweigungen abgeben. Der Körper dieser Zellen ist manchmal so unregelmässig, dass die protoplasmatische Masse um den Kern herum geringer ist, als an den zu Fortsätzen geformten Theilen. Das Protoplasma ist im Allgemeinen reichlich, homogen, hyalin und färbt sich nach der Weigert'schen Methode mehr oder weniger stark blau. Manchmal ist ein einziger Kern vorhanden, manchmal sind zwei oder drei Kerne da von granulirtem Aussehen, welche sich stärker blau färben, als das Protoplasma.

Diese Zellen fand ich z. B. bei oinem Gliom der weissen Substanz des rechten Frontallappens (Zaniboni) zahlreich. Bei diesem Gliom bildeten sie den wichtigsten Theil der Geschwulst und waren innerhalb eines dichten Netzes von Neuroglia-Fäserchen vertheilt (Taf. XIV Fig. 3).

4. Unregelmässige Zellen von mittlerer Grösse, welche mit kurzen und dicken, nicht verzweigten Fortsätzen versehen sind. Ihr Protoplasma färbt sich schwach blau in den centralen Theilen, wo der Kern sich findet, ein wenig stärker in der Peripherie, aus der die Fortsätze entstehen. Ier Kern färbt sich schwach und ist vom Protoplasma nicht gut differenzirt. Diese Zellen werden oft von Neuroglia-Fäserchen durchbrochen, welche in 
den Präparaten nach Weigert eine stark blaue. Färbung zeigen.

5. Rundliche, 12-18 $\mu$ grosse, platte Zellen, ohne Fortsätze, mit reichlichem, homogenem, hyalinem Protoplasma, mit einfachem oder doppeltem, kleinem, gegen das Centrum gelegenem Kerne. Diese Zellen erinnern an die endothelialen und gehen im Allgemeinen kein intimes Verhältniss mit den Neuroglia-Käserchen ein, sondern sind zwischen gewissen, von den genannten Fäserchen begrenzten Räumen (Fall Baessato) eingelagert (Taf. XIV Fig. 4).

6. Grosse, unregelmässig drejeckige, polygonale Zellen mit sehr reichlichem Protoplasma, welches sich nach der Weigertschen Methode blau färbt und in innige Beziehungen zu den Neuroglia-Fäserchen an der Peripherie tritt. Die Fäserchen sind dem Zellkörper dicht angelagert, und sie entfernen sich davon, wie Fortsätze, neben den Ecken, indem sie sich mit den von der entgegengesetzten Seite kommenden Fäserchen vermischen. Die Fortsätze sind offenbar keine ächten Protoplasma-Ausläufer, wie die der oben unter No. 3 beschriebenen Zellen, denn sie haben eine ganz andere Färbungs-Reaction, als jene. Man muss, mit Kölliker annehmen, dass dieZellen auch unter pathologischen Zuständen aus zwei Schichten, einer centralen und einer peripherischen, bestehen; die peripherische Schicht tritt mit den Neuroglia-Fäserchen in Verbindung (Taf. XIV Fig. 5).

Solche Zellen habe ich bei den Rinden-Gliomen (BaessatoAgostini) gefunden und ferner in einem Gliom, welches EpithelVerlagerungen zeigte (Cervesato, ein 5 jähriges Kind). Meiner Meinung nach sind diese Zellen den von Buchholtz gesehenen ganz ähnlich. Sie würden eine Rückkehr zu dem embryonalen Zustand darstellen, welche diese Elemente denen des Ectoderms, von welchen sie sich ableiten, gleich machen würde. Diese Zellen, welche ich "gliogene" nennen möchte, unterscheiden sich von den eben beschriebenen (No.5) sehr wenig, nur durch das Verhältniss des peripherischen Theiles zu den Neuroglia-Fäserchen. Wir haben es wahrscheinlich mit Zellen derselben Qualität zu thun, abgesehen davon, dass die letztgenannten Elemente reifer, älter waren, und dass ihr Protoplasma vielleicht 
jene Entwicklungsphase erreicht hat, in welcher es geeignet ist, Neurogliafäserchen zu bilden.

7. Zellen von verschiedener Grösse und Gestalt, die meistens unregelmässig verlängert sind, bei welchen der granulirte, stark färbbare Kern überwiegt, während das Protoplasma sehr gering und kaum sichtbar ist. Fortsätze sind nicht vorbanden, auch die Beziehungen dieser Zellen zu den Fäserchen sind nicht deutlich. Diese Zellen sind in gewissen Theilen der Gliome sehr zahlreich, so dass sie dicht an einander gedrängt sind. Wo sie zerstreut liegen, ist die intercelluläre Substanz homogen, fein granulirt, nach der Weigert'schen Methode nicht färbbar, und zeigt nur wenige Fäserchen an den peripherischen Theilen der zelligen Anhäufungen, welche man deshalb mit sarcomatösen Zellen verwechseln könnte. Indem sich diese Zellen an einander drängen, bilden sie Knötchen, die durch Neuroglia-Fäserchen ziemlich gut begrenzt sind; letztere erzeugen dichte Geflechte um die Knötchen herum (Taf. XV Fig. 2).

\section{V.}

Verhältaisse der Zellen zu den Fäserchen bei den Neubildungen der Neuroglia.

Das Neuroglia-Gewebe bildet, wie bekannt, den Schutzapparat der Nerven-Elemente, d. h. der Nervenfasern und der Ganglienzellen, wie auch der Blutgefässe, welchen die ErnährungsFunction der Nervensubstanz obliegt. Dasselbe besteht aus eigenen Zellen und aus einem System von sehr dünnen Fäserchen, die sich nach verschiedenen Richtungen verbreiten und ein sehr zartes Netz bilden, in dessen Mitte die Neurogliazellen verstreut liegen. Dieses Netz erscheint nach der Meinung einiger angesehener Beobachter ( $W$ eigert) als das wichtigste Element der Intercellularsubstanz; es ist aber wohl nicht auszuschliessen, dass auch noch andere Zwischensubstanzen sich in den Nervencentren finden.

Ueber die Entstehung dieser Fäserchen können wir mit Sicherheit nur wenig aussagen; über ihre Function und ihre Anordnung stimmen die Autoren nicht überein, was meistens von den technischen Methoden abhängt, die für das NeurogliaStudium angewendet worden sind. 
Seit Frommann $\left.n^{1}\right)(1862)$ und Deiters $\left.{ }^{2}\right)(1877)$, welche, wie es scheint, die ersten waren, die mit der Carmin-Färbungsmethode die Fäserchen entdeckten, aus denen die Neuroglia sich zusammensetzt, bis vor fünf Jahren, gab es eine grosse Reihe von Histologen, (Jastrowiz ${ }^{3}$ ), Boll ${ }^{4}$ ), Kölliker ${ }^{5}$ ), Golgi ${ }^{6}$ ), Gierke ${ }^{7}$ ) u. A.), welche die Fäserchen als ein unmittelbares Product der Neurogliazellen betrachteten; die Fäsarchen sollten aus den Fortsätzen der Zellen selbst entstehen. Wir müssen aber Frommann das Verdienst zuerkennen, diese Fäserchen als ganz unabhängig von dem Bindegewebe der weichen Hirnhaut beobachtet zu haben.

Sehr wichtig in dieser Beziehung waren die von Golgi im Jahre 1871 nach einer besonderen Methode vorgenommenen und bis 1884 fortgesetzten Untersuchungen. Diese hatten den Zweck, die Beziehungen zwischen den Fäserchen und den Neurogliazellen festzustellen.

Jastrowitz erfand den Namen "Spinnenzellen“, um die Neuroglia- oder Deiters'schen Zellen zu bezeichnen, und gab damit deutlich der Ansicht Ausdruck, dass jene Zellen Fortsätze haben.

Gierke und Kölliker, sowie Andere stellten sich die Neurogliazellen vor als versehen mit Fortsätzen, aus welchen die Neuroglia-Fäserchen entstehen. Den Folgerungen dieser Beobachter setzten aber andere Histologen abweichende Meinungen entgegen. Schon Boll hat den differenzirten Charakter der

1) Frommann, Untersuchungen über die normale und pathol. Anatomie des Rückenmarkes, 1. Theil, 1864.

2) Deiters, Untersuchungen äber Gehirn und Räckenmark des Menschen and Säugethieres. Braunschweig, 1865 .

3) Jastrowitz, Ueber Encephalitis und Myelitis im ersten Kindesalter. Archiv für Psychiatrie, Bd. 2, S. 389.

4) Boll, Die Histologie und die Histogenese der nervösen Centralorgane. Archiv f. Psychiatrie, Bd. 4, 1874.

5) Kölliker, Handbuch der Gewebelehre des Menschen, Bd. 2, 1893, und Traité d'embryologie, 1882.

6) Golgi, Contributo allo studio della fine anatomia degli organi centrali del sistema nervoso. Rivista clinica, Bologna, 1871.

7) Gierke, Die Schutzsubstanz des Central-Nervensystems. Archiv für mikroskopische Anatomie, Bd. 25, S. 441. 
- Neuroglia-Fasern hervorgehoben; indem er die Neurogliazellen mit denen des embryonalen Bindegewebes vergleicht, bestätigt er, dass die Zellen bei dem einen, wie bei dem anderen Gewebe nichts anderes darstellen, als das aus einer grossen Menge von gut differenzirten Faseru bestehende Centrum, dem sich die Fasern entweder von einer oder von allen Seiten her nähern; bei dem einen, wie bei dem anderen Gewebe besitzt die Zelle einen Kern, welcher meistens von einer geringen Masse granulirter Substanz umgeben ist; endlich ist es bei dem einen, wie bei dem anderen noch unbestinmt, ob diese granulirte Substanz, welche den Kern umgiebt und das Maschenwerk der Fäserchen enthält, als lebendes und functionirendes Protoplasma, oder als eine eiweisshaltige und formlose Substanz zu betrachten sei.

Der letzte aber und entscheidende Schritt in dieser Frage wurde von Ranvier ${ }^{1}$ ) gemacht, welcher an Präparaten vom Rückenmark Erwachsener bewies, dass die sogenannten Fortsätze der Neuroglia- oder Deiter's'schen Zellen nicht, wie Frommann und nachher die Anderen dachten, Ausstrahlungen des Protoplasma-Körpers, sondern gut differenzirte Fäserchen sind, welche durch den Zellenkörper gehen oder sich auf denselben stützen. Dieselben verbreiten sich vom Zellkörper aus nach allen Richtungen, aber die den Zellkörper bildende Substanz setzt sich nicht längs der Fäserchen fort und scheint aus einem Material zu bestehen, welches sich von der den Fäserchen entsprechenden Substanz chemisch unterscheidet. Im embryonalen Stadium sind die normalen Neurogliazellen genau sternförmig und die Zellfortsätze einfache Verlängerungen des Zellkörpers. Später kommt erst die Differenzirung der Fasern von dem Körper zum Vorschein. Diese Anschauungen Ranvier's wurden dann weiter von Weigert entwickelt, welcher die Richtigkeit derselben mit grosser Deutlichkeit bestätigte. Er wendete zum Studium der Neuroglia eine wirklich originelle Färbungs-Methode au, mittelst deren er sehr elegante und beweiskräftige Präparate zu Stande bringen konnte.

I) Ranvier, De la névroglje. Comptes rendus de l'Academje des sciences, 5. juin 1882. - De la névroglie, Archives de Physiologie normale et pathologique, 1883 , Tom I: 
Nach $\mathrm{ihm}^{1}$ ) dringen die Fäserchen durch die Zelle bis in die Nähe des Kernes und durchlaufen ein durchsichtiges Protoplasma, welches sich mit der von ihm angegebenen Methode nicht färbt; einige dieser Fäserchen gehen in gerader Richtung bis an die entgegengesetzte Seite der Zelle, andere dagegen biegen in der Nähe des Kernes mit mehr oder weniger scharfem Bogen ab, andere endlich unterbrechen sich plötzlich und hören neben dem Kerne auf (abgeschnittene und im optischen Durchschnitte beobachtete Fasern). Uebergänge der Fasern in dieses Protoplasma sind nicht bemerkbar; man darf sogar dieselben als chemisch verschiedene Bildungen betrachten.

Der Autor stellt folgende Schlüsse auf:

1. Die Neuroglia-Fasern, welche bis jetzt für Fortsätze der Deiters'schen Zellen gehalten worden sind, sind chemisch nicht mit dem Protoplasma identisch.

2. Diese Verschiedenheit bemerkt man schon innerhalb des Zellkörpers in der Nähe des Kernes, und dieselbe wird nicht stärker, wenn die Fasern sich nach und nach von dem Zellkörper entfernen.

3. Die meisten sogenannten Zellfortsätze sind in der That gar nicht Fortsätze, sondern nur Fasern, die von dem Protoplasma vollständig differenzirt worden sind.

Von den Kernen oder den zelligen Elementen, denen solche Kerne angehören, entsprechen nur diejenigen den Deiters'schen Zellen, welche mit den gewöhnlichen strahlenförmigen Fäserchen in einer typischen Weise in Verbindung stehen.

Es giebt also eine beträchtliche Zahl von Kernen zwischen den Fibrillen, in deren Nähe die Fäserchen unregelmässig verlaufen; diese sind als Neuroglia-Kerne zu betrachten.

Die Deiters'schen Zellen wären also nach dem durch Weigert's classische Färbungs-Methode dargebotenen Beweise nichts Anderes, als scharf von den Nervenzellen differenzirte Neurogliazellen; sie würden kleine Zwischenräume des Neuroglia-Netzes darstellen, bei welchen die Fäserchen nicht in Continuität, sondern nur in Berührung mit den Zellen stehen würden.

Demnach wäre das System der.Neuroglia-Fäserchen, welche sich um oder innerhalb der Zellen verbreiten, nichts Anderes,

1) Weigert, Beiträge zar Kenntniss der normalen menschlichen Neuroglia. Frankfurt a. M., 1895. 
als eine Art von intercellularer Substanz. Nach Weigert ist es absolut auszuschliessen, dass die letztere eine Nervensubstanz sei: erstens, weil die nervösen Elemente nach seiner Methode immer ungefärbt bleiben, während die Fäserchen sich tiefblau färben; zweitens, weil sie nicht aus demselben protoplasmatischen Material, wie der Zellkörper, sondern aus einer veränderten, nicht mehr protoplasmatischen Substanz bestehen; drittens, weil die Fäserchen und die entsprechenden Zellen sich in pathologischen Zuständen genau wie ein Bindegewebe verhalten, d. h., sich vermehren, wenn das nervöse. Element zu Grunde geht. Diese letztere Annahme Weigert's müssen wir nicht in dem Sinne auffassen, dass die Zerstörung des specifischen Nervengewebes der Neubildung der Neuroglia immer vorangeht, vielmehr ist zuzugeben, dass die beiden Erscheinungen sich oft gleichzeitig entwickeln. Es kommt wirklich nicht selten vor, dass man Nervenfäserchen und Ganglienzellen innerhalb der gliomatösen Neubildungen findet.

Die oben dargelegten Meinungen, welche aus den Untersuchungen Ranvier's und Weigert's bervorgehen, widersprechen absolut den Theorien der ersten Beobachter, und der Grund dieses Widerspruches liegt vielleicht in der Verschiedenheit der technischen Methoden, welche angewendet worden sind, um die Stützzellen und die entsprechenden Fäserchen deutlich darzustellen und dieselben von den specifischen Nerven-Elementen zu unterscheiden. Da nun viele Eigenschaften bezüglich der Structur und Entstehung der Neuroglia, welche von den Histologen, die sich mit dieser Frage bis jetzt beschäftigt haben, bemerkt worden sind, so ist anzunehmen, dass sich wichtige entsprechende Verhältnisse auch bei den pathologischen Erscheinungen finden; und da aus der Literatur der letzten fünf Jahre nicht. hervorgeht, dass jemand die Eigenthümlichkeiten der Structur und der Histogenese der pathologischen Neuroglia studirt und zu erklären versucht hat, so halte ich es für nothwendig, über einige von mir beim Studium von etwa zwölf Gliomen gefundene Eigenschaften zu berichten. Diese beziehen sich besonders auf die Art und Weise, wie sich die Fäserchen verhalten und entstehen, und auf ihre Beziehungen zu den Neurogliazellen und den Blutgefässen. 
VI.

Ueber das Verhalten der Neuroglia-Fasern und ihre Beziehungen zuden Neuroglia-Zellen in pathologischen Zuständen.

Eine Frage, die sich dem die Structur der Neuroglia Studirenden zuerst darbietet, betrifft die Verhältnisse, welche zwischen den Fäserchen und den neugebildeten Zellen herrschen, und da diese, besonders bei den gliomátösen Neubildungen, oft Eigenschaften annehmen, welche sich von denen normaler Zellen in Bezug auf die Form, oder auf die Grösse, oder auch auf die Zahl und die Färbungs-Fähigkeit ihres Protoplasma unterscheiden, so ist es sehr interessant, nachzuforschen, wie die Fäserchen sich zu denselben verhalten.

Schon oben habe ich bemerkt, wie die Zellen, welche als ächte pathologische Neubildungen zu betrachten sind, sich im Allgemeinen von den normalen Zellen viel unterscheiden. Bei den Gliose-Heerden aber viel deutlicher, als bei den Gliomen, findet man neben grossen, rundlichen und unregelmässig polyedrischen Zellen mit reichlichem, fast durchsichtigem und nach Weigert's Methode schwach färbbarem Protoplasma, kleine Zellen mit rundem, stark färbbarem Kerne, der von einem dünnen, durchsichtigen, nicht färbbarem Protoplasma-Mantel umgeben ist. Diese Zellen entsprechen denjenigen der normalen Neuroglia und haben keine wirklichen Fortsätze; die Fäserchen der intercellularen Substanz dringen durch die dünne Protoplasma-Schicht und gehen manchmal von der einen zur entgegengesetzten Seite, manchmal liegen sie bogenartig und treten wieder neben dem Eintrittspunkte aus. Diese Fäserchen sind nicht mit den protoplasmatischen Fortsätzen zu verwechseln.

Dieselbe Art und Weise, sich zu verhalten, zeigen die Fäserchen der pathologischen Neuroglia, wie ich bemerken konnte, noch bei anderen Zellenformen, die von mir in verschiedenen Gliomen und Gliosen gefunden worden sind, und welche den in No. 3 und 4 beschriebenen Zellen besonders entsprechen. Sie sind gross und unregelmässig polyedrisch, mit deutlichen, konischen und wellenförmigen Protoplasma-Fortsätzen, welche sich nur schwach nach Weigert's Methode färben. Das Protoplasma dieser atypischen und für die pathologische Neuro- 
glia eigentlichen Zellen erscheint oft von Fäserchen durchschnitten, welche nach verschiedenen Richtungen, bald der Länge nach, bald quer hindurch gehen, indem sie neben dem Kerne vorbei gehen (Taf. XIV Fig. 6).

Solche Fäserchen treten sehr deutlich innerhalb der Masse der protoplasmatischen Substanz hervor, weil sie sich bei den nach der Weigert'schen Methode gefärbten Präparaten schwarzblau färben, und sich sehr leicht von den protoplasmatischen Fortsätzen unterscheiden, welche aus dem Zellkörper hervorragen.

Inmitten des Protoplasma bemerist man manchmal runde, granulirte Bildungen, welche uns an Nebenkerne erinnern, die aber nichts Anderes sind, als quer geschnittene und im optischen Schnitt beobachtete Bündelchen. Bei einigen Gliomen der Hirnrinde (Fall Baessato), wo die Zellelemente sehr zahlreich, gross, unregelmässig sind, und wo um dieselben eine geringe farblose Grundsubstanz erscheint, in deren Mitte ein dichtes, mit der Weigert'schen Methode stark blau gefärbtes Fasernetz hervortritt, finden sich die Fäserchen in ziemlich innigem Verhältniss zu dem Zellkörper; sie bleiben aber von der Protoplasma-Substanz getrennt und vermischen sich nicht mit den Fortsätzen des Zellkörpers, wenn auch ihre Form sehr unregelmässig sein kann. Solche Fäserchen gehen entweder durch den Protoplasma-Körper, mehr oder weniger in der Nähe des Kernes, oder sie sind so innig mit der Oberfläche der Zelle selbst verbunden, dass man sich an die Hypothese Kölliker's erinnern kann, nach welcher das Protoplasma der Neurogliazelle aus zwei verschiedenen Theilen besteht, aus einem centralen, der in Verbindung mit dem Kerne steht, und aus einem peripherischen, welcher mit den Fäserchen zusammenhängt.

Nach der Richtung des Schnittes, von dem der Zellkörper betroffen wurde, gehen die meistens an der Peripherie des Zellkörpers gelegenen Fäserchen der Länge nach durch den Rand der Zelle, oder sie erscheinen im Querschnitte als eine punktförmige, schwarzblau gefärbte Masse.

Dieser Befund scheint mir von grosser Wichtigkeit zu sein, indem er zur Erklärung der Entstehung der Neuroglia-Fäserchen auch bei pathologischen Zuständen beiträgt. Auch hier wären 
solche Fäserchen ein Product der protoplasmatischen Substanz, welche sich chemisch verändert hat.

Da nun das System der Fäserchen, welche das NeurogliaNetz bilden, vielmehr eine Art von intercellularer Substanz darstellt, als das Resultat der Verzweigung von Zellprotoplasma-Fortsätzen, so darf man annehmen, dass es Zellen auch in pathologischen Zuständen giebt, welche, wenn sie eine bestimmte Phase ihrer Entwicklung erreicht haben, zur Bildung einer besonderen intercellularen faserigen Sub$\operatorname{stan} z$ mitwirken.

Damit verhalten sich die Neurogliazellen, obwohl sie immer Sprösslinge des Ectoderms bleiben, genau wie die Zellelemente des Bindegewebes.

Zur Bestätigung der Behauptung, dass einige der Fäserchen aus dem veränderten Zellprotoplasma entstehen, dient als vollgültiger Beweis die Thatsache, dass viele Fäserchen innige Beziehungen zu dem Körper der Neurogliazellen haben. Es ist aber nicht als ausgeschlossen anzusehen, dass ein Theil der Fäserchen ausserhalb der Zellen sich bilden kann.

Was nun an den von mir beim Studium der Gliome beobachteten Erscheinungen wichtig und bemerkenswerth scheint, ist die Beobachtung, dass die Zellen nicht an allen Theilen des Gliomes dieselben Beziehungen zu den Füserchen der Grundsubstanz haben.

Man findet manchmal in der That bei den Präparaten einiger Gliome rundliche Zonen, welche mehr jungen Knötchen entsprechen (Agostini'scher Fall), deren Grenze, während sie an frischem Materiale nicht ganz gut bezeichnet war, bei den nach Weigert's Methode gefärbten Präparaten als eine bläuliche, die blassere Zone des jungen Knötchens umgebende Linie erscheint. Die histologische Untersuchung beweist also, dass die Zellelemente bei diesen Zonen im Ueberfluss vorhanden sind, und diese Elemente erscheinen mit eiförmigen oder unregelmässigen, grossen Kernen, welche mit geringem, durchsichtigem Protoplasma versehen und durch eine formlose Zwischensubstanz getrennt sind, in der man nur sehr spärliche und dünne Fäserchen beobachtet (Taf. XV Fig. 2a). Die Zellen stehen absolut in keiner Verbindung mit den Fäserchen, die man in der Grundsubstanz 
beobachtet hat; diese letzteren liegen weit von den Zellen ab und sind ganz gewiss nur von dieser Grundsubstanz mittelst einer besonderen Differenzirung, welchedurch chemische Veränderungen verursacht worden ist, abzuleiten. Es ist aber auch denkbar, dass eine gewisse Zahl dieser Fäserchen aus dem Protoplasma der Neurogliazelle sich bilden. Und da man an einigen Stellen gewisser Gliome und genau, wo der Zuwachs der Geschwulst geschieht, embryonale, den Spongioblasten ähnliche Zellen fast ausschliesslich findet, während die Fäserchen entweder fehlen oder in sehr geringer Zahl vorhanden sind, so ist es anzunehmen, dass die Bildung der Fäserchen von den Zellen nur in einem bestimmten Entwickelungs-Stadium der Zellen selbst stattindet.

Seit $1890^{1}$ ) ist es auch Weigert gelungen, Gliome zu finden, bei welchen Zellen im Ueberfluss vorhanden waren, während keine oder sehr spärliche Fäserchen erschienen. Er hatte Gelegenheit, zwei Gliome zu untersuchen, bei welchen er mit grossem Erstaunen keine Fäserchen an der Dicke der Geschwulst fand, während dieselben mit prächtiger Färbung bei dem umgebenden Gewebe erschienen.

Weigert wollte aber noch nicht eine entscheidende Erklärung des Befundes geben. Zuerst dachte er, dass es sich um eine Unvollkommenheit der Färbungsmethode in diesem Falle handelte, später stellte er die Hypothese auf, keine ächten Gliome gefunden zu haben; ein drittes Mal nahm er an, dass der untersuchte Vorgang eine Neubildung der Neuroglia wäre, und dass die Zellen nicht die Eigenschaft hätten, Fäserchen zu bilden und sich wie Sarcomzellen zu verhalten. Da er nun keine von diesen Bedingungen bestätigen konnte, hielt er es endlich für wahrscheinlich, dass die Fäserchen in einer zweiten Periode zu Grunde gegangen wären.

Dieselben Betrachtungen könnte ich selbst machen, obwohl es meinen Präparaten nach als unwahrscheinlich gelten sollte, dass es sich nicht um ein gliomatöses Gewebe hier handelte oder dass die Fäserchen zufällig verschwunden waren.

1) Weigert, Zur pathologischen.Histologie des Neurogliagerüsts. Centralblatt für allg. Pathol. u. pathol. Anatomie, No. 23, 1890. 
Wenn wir auch nicht leugnen können, dass es möglich sei, durch die Anwendung verschiedener anderer Färbungs-Methoden in der intercellularen Substanz des jungen gliomatösen Gewebes die Fäserchen deutlich $\mathrm{zu}$ machen, welche mit der ausgezeichneten Weigert'schen Methode, mittelst deren die Fäserchen der normalen Neuroglia und der älteste Theil des gliomatösen Gewebes sehr deutlich dargestellt werden können, nicht sichtbar sind, so darf man wohl vermuthen, dass die Fäserchen-Bildung bei der Entstehung des gliomatösen Gewebes der Zell-Entwicklung folgt. Und da die Fäserchen sich wie eine intercellulare Substanz verhalten, ist es uns bis zu einem gewissen Punkt erlaubt, die Masse der Fäserchen als ein Secretions-Product der Neurogliazellen zu betrachten. Welche Entwicklungs-Phase dieser zelligen Elemente mehr geeignet sei, die Fäserchen zu bilden, und welche Bedingungen solche Bildung begünstigen, ist gar nicht leicht zu sagen. Man darf aber wohl daran denken, dass die Neurogliazellen, welche sich dem Embryonaltypus, d. h. dem Typus der ectodermischen Elemente mehr nähern, nicht die Eigenthümlichkeit haben, Fäserchen zu bilden. Diese Eigenthümlichkeit gehört vielmehr dem Protoplasma jener Zellen, welche eine gewisse Entwickelungsphase schon erreicht haben.

Dies findet, wie es scheint, auch bei der Bildung der normalen Neuroglia statt. Diese Frage wollen wir jetzt aber nicht beachten; wir werden uns damit in einer anderen Arbeit beschäftigen.

In Bezug auf das, was wir nun über die Entstehung der Fäserchen gesagt haben, so können wir, wenn wir daran denken, dass dieselben nur bei einer bestimmten Phase des zelligen Lebens erscheinen, uns erklären, wie es möglich ist, die jungen Gliome, welche aus Embryonal-Elementen bestehen, mit einem Sarcom zu verwechseln.

Diese Verwechslung zwischen den beiden Geschwulstformen wird noch leichter, da einige Eigenschaften, die von Gowers, Ströbe u. A. angenommen wurden, um Gliome von Sarcomen zu unterscheiden, nicht constant sind. In der That hat das Gliom nicht immer ein peripherisches, nach der umgebenden Nervensubstanz infiltratives Wachsthum, sondern manchmal sieht 
diese Entwickelung genau so aus, wie bei den Sarcomen, d. h. die umgebende Nervensubstanz wird von einer dehnbaren Entwickelung rundlicher Knötchen durchdrungen.

Aber die Anwesenheit eines Entartungs- oder Erweichungszone an der Nervensubstanz, welche von Gowers in seinem Handbuch als ein Unterschieds-Charakter für die Sarcome an genommen wurden, hat sich um die sarcomatösen Knötchen herum nicht constant gezeigt.

Andererseits gilt das aus dem speciellen Verhältnisse zu der von dem sarcomatösen, aber nicht von dem gliomatösen Gewebe durchwachsenen weichen Hirnhaut entnommene Kriterium nur für die oberflächlichen Geschwülste, und auch nicht für jede Art von Gliomen; denn es ist mir gerade vorgekommen, wie ich weiter ausführen werde, wenn ich von der Topographie der neugebildeten Glia sprechen werde, einen Fall von verbreiteter Gliose der Vierhügel, der oberen Kleinhirnschenkel (Bindearme) und des höheren Theiles des Wurmes (Lingula und Culmen) und auch der lateralen Massen (Lobulus centralis) zu finden, wo die Fäserchen-Bildung nicht nur im Innern der cerebellaren Windungen beschränkt war, sondern auch die oben liegende Pia mater durchdrang. Innerhalb des dichten und zartesten Fibrillennetzes beobachtete man nur sehr spärliche Neurogliazellen (Taf. XV Fig. 3), so dass man auch wegen dieses histologischen Befundes sofort ausschliessen konnte, dașs es sich um eine sarcomatöse Neubildung handelte.

Ein weiterer Differenzirungs-Charakter zwischen den Gliomen und Sarcomen, welchen man nach dem Befund des ïbrig gebliebenen, an den centralen Theilen des Sarcomes wahrscheinlich nicht befindlichen Materials der markhaltigen Fasern oder der Ganglienzellen finden kounte, hat einen Werth nur für einige Formen von Gliomen.

Was nun die anderen Structur-Eigenschaften der Fäserchen in der pathologischen Neuroglia anbelangt, so muss ich gleich Folgendes bemerken: Bei den von elf verschiedenen GliomFormen der verschiedenen Theile der Cerebrospinal-Achse, sei es mit der Mallory'sclsen, sei es mit der Golgi'schen, sei es endlich mit der Weigert'schen Methode hergestellten Präparaten, erscheinen die Fäserchen viel besser individualisirt und diffe- 
renzirt mit dieser letzten Methode, als mit der Golgi'sçen schwarzen Reaction. - Die Dicke solcher Fäserchen ist im Allgemeinen grösser, als die der Füserchen der normalen Neuroglia. Ihre Dicke ist nicht bei dem ganzen Verlauf gleich, sondern man bemerkt verlängerte Anschwellungen an gewissen Punkten des Fäserchens, so dass das Fäserchen spindelförmig aussieht, während man an anderen Stellen fast dreieckige Anschwellungen beobachtet, welche aus einer Seite des Fäserchens hervorragen und mit kleinen, von dem Fäserchen selbst durchschnittenen Neurogliazellen nicht zu verwechseln sind.

Man darf wohl ausschliessen, dass es sich hier um Zellen handelt, denn es erscheint keine differenzirte Substanz um das Fäserchen herum, welche als protoplasmatisches Material zu betrachten wäre. Wir können es ebenso für ausgeschlossen halten, dass diese Anschwellungen den Intersections- oder Berührungs-Punkten von zwei oder mehreren Fäserchen entsprechen. Solche Anschwellungen der Neurogliafasern wurden schon von Weigert an der Hirnrinde einiger von progressiver Paralyse afficirter Individuen beobachtet. Diese Verdickungen oder Vergrösserungen erscheinen an dem Biegungspunkt des Fäserehens, manchmal auch längs des geradlinigen Verlaufes. Neben diesen gröberen, mit beschränkter A usdehnung versehenen Fäserchen sieht man (bei den vorgerückten Gliosen und Gliomen) auch dünnere und dünnste Fäserchen, welche sich manchmal parallel anordnen und Bündelchen von drei, vier Fibrillen bilden, die sich mit den anderen dickeren Fäserchen kreuzen. Auf solche Weise entstehen oft zarte Geflechte, die aus einigen Lagen bestehen. Die Fäserchen gehen manchmal in gerader Richtung, manchmal liegen sie bogenartig, manchmal sind sie wellenförmig; einige sind kurz, andere lassen ihre Enden gar nicht sehen, sei es, weil sie sich nach langem Verlauf biegen und deshalb bei der Section des Stückes abgeschnitten werden, sei es, weil sie sich an ihren Enden über die benachbarten Fäserchen legen. Man kann also vermuthen, dass die Bahn dieser Fäserchen sehr veränderlich ist. Das geschieht ebenso oft bei den Gliomen und Gliosen, welche nur spärliche Zellen haben, wie bei denjenigen, wo die Zellelemente im Ueberfluss vorhanden sind. Auch bei den nach Golgi erhaltenen Präparaten ist es unmög- 
lich, eine grössere Helligkeit der Bilder zu bekommen, um die Bahn der Fäserchen besser bestimmen zu können.

Bei diesen Präparaten erscheinen die Fäserchen gröber und zeigen sehr häufig Anschwellungen, welche sich schwarz färben. Da es nun, wenn der Zellkörper sich schwarz färbt, unmöglich ist, irgend eine Differenzirung, welche uns die Fäserchen deutlich zeigt, im Inneren desselben zu bemerken, so scheint es, dass viele von jenen Fäserchen sich innerhalb der Zellen erschöpfen.

Bei den nach Weigert's Methode erhaltenen Präparaten dagegen zeigen sich die Fäserchen im Innern des Zellkörpers gut differenzirt, und es ist möglich, zu bemerken, wie dieselben manchmal plötzlich anhalten, weil sie quer oder schief geschnitten worden sind, während sie manchmal als blaue Pünktchen beim Zellkörper erscheinen.

Offenbar sind diese blauen Pünktehen nichts Anderes, als im optischen Schnitte gesehene Fäserchen.' Solche Pünktchen sind meistens neben der Peripherie des Zellkörpers gelegen. - Die Fäserchen sind solide Bildungen, sie zeigen also im Innern keinen Hohlraum; das gilt auch für jene Stellen, wo die Fasern gröber oder mit Anschwellungen versehen erscheinen. Die Oberfläche der Fäserchen bei den nach Weigert's Methode hergestellten Präparaten ist glatt, regelmässig, ohne Hervorragungen oder dornartige Fortätze, während sie bei den mit Golgi's Methode erhaltenen Präparaten oft moosartige Rauhigkeiten zeigt. Wenn bei diesen Präparaten die Reaction gut gelungen ist, haben viele Fäserchen, die als Zellfortsätze erscheinen, eine ungleiche Dicke, und man kann nicht sicher sagen, ob die dickeren Fasern durch Niederschlag der Silbersalze auf zwei benachbarte Fäserchen entstehen.

Bei einem typischen Gliom der Rinde der ersten und zweiten linken Stirnwindung (Baessato), das ich mit der Golgischen und Weigert'schen Methode zum Vergleich untersuchte, habe ich bemerkt, dass die Bilder bei den mit der letzten Methode erhaltenen Präparaten im Allgemeinen deutlicher waren, und dass namentlich auch die zartesten, zwischen den Zellen gelegenen Fäserchen gut differenzirt erschienen, während dieselben Fäserchen bei den nach Golgi'scher Methode erhaltenen Präpa- 
raten durch eine Niederschlagsmasse zusammengeschmolzen schienen. Manchmal ergab sich aus dieser Verschmelzung das Bild canalisirter, zwischen den Geschwulstzellen verbreiteter Fortsätze, welche, da diese Zellen sehr zahlreich waren, oft dicht bei einander lagen. In Folge dessen schien es an einigen Präparat-Abschnitten, als ob die Gliomzellen vermittelst eines Systems dünner Canälchen oder Ausläufer verbunden waren, welche den Zellen eine unregelmässig vielstrahlige Form gaben.

In zahlreichen, von mir nach der Weigert'schen Methode erhaltenen Präparaten erschienen die Fäserchen immer homogen schwarzblau gefärbt. Nur bei einem Falle von weichem, myxomatösem Gliom des Thalamus opticus eines 13 jährigen Mädchens beobachtete ich, dass die Fäserchen sich an den weichsten Theilen des Glioms schwer färbten, dass sie granulirt und hier und da wie fragmentirt erschienen, und dass ihre Beziehungen zu den Zellen nicht recht klar waren. Dieses besondere Aussehen der Fäserchen war aber dem sogenannten granulirten Zerfall zuzuschreiben, welcher von dem-Oedem und dem Nekrobiose-Zustande des Glioms abhing. Die Geschwulst erschien in der That auch makroskopisch blassgrau gefärbt, halb durchsichtig und weich, wie ein Myxom.

Ob dieses Gewebe Mucin enthielt, konnte ich nicht bestimmt sagen; dagegen schien es mir möglich, auszuschliessen, dass es sich um eine cadaveröse Erweichung handelte.

Der granulirte Zerfall, welchem die Fäserchen bei gewissen Gliomen wegen des Oedems und der Nekrobiose (die sogenannten Myxogliome einiger Autoren) unterworfen sind, dient als Hülfsmittel für den Nachweis der Neuroglia-Natur dieser Fäserchen, welche den widerstehenden Theil des neugebildeten Gewebes ausmachen, und ist ferner ein Unterscheidungs-Merkmal anderen Bildungen gegenüber (namentlich dem Fibrin), mit denen sie wegen ihrer Eigenschaft, ein Netzwerk zu bilden und sich nach derselben Methode zu färben, wie die Neuroglia, leicht zu verwechseln sind. Es ist in der That bekannt, dass Fibrin an der Peripherie einiger Sarcome oder Gliome, namentlich von der die Geschwulst umgebenden Substanz, gebildet werden kann.

In dem Falle eines von mir studirten Endothel-Sarcoms, welches an der zweiten rechten Occipitalwindung einer 48jährigen 
syphilitischen Frau (Zago Maria, 28. 12. 99) entstanden war, wurde eine solche Fibrin-Bildung durch einen Bluterguss verursacht, welcher sich in der weissen, subcorticalen Substanz befand. Nach dem Verschwinden der meisten rothen Blutkörperchen bildete sich eine weissgelbliche, weiche, halb durchsichtige, etwa $1 \mathrm{~cm}$ dicke Zone rings um die neoplastische Masse. In den nach der Weigert'schen Methode für die Neuroglia erhaltenen Präparaten bestand diese Schicht aus groben Fäserchen, welche so mit einander anastomosirten und sich kreuzten, dass sie zuweilen sehr kleine Räume begrenzten, in denen man Reste von rothen, in kleinen Gruppen vereinigten Blutkörperchen und auch Leukocyten beobachtete. Diese Eigenschaften stellten die Annahme ausser Zweifel, dass es sich um Fibrin handelte.

Keines von diesen Fibrin-Trabekeln zeigte, obwohl sie mit transsudirter Flüssigkeit in Berührung standen, jenen granulirten Zersetzungszustand, welchen die Fäserchen des erweichten Gliomes hatten, von dem ich oben gesprochen habe.

Die Leichtigkeit, mit welcher die Neuroglia-Fäserchen, sei es wegen der cadaverösen Erweichung, sei es wegen des Oedemes oder der Nekrobiose des Gliomes, von einer granulirten Zersetzung betroffen werden können, muss also als charakterisches Merkmal für die Unterscheidung von Fibrin-Trabekeln und NeurogliaFasern in den nach Weigert hergestellten Präparaten gelten.

Die Neuroglia-Fasern anastomosiren weder mit einander, noch verzweigen sie sich, sondern sie legen sich über einander, indem sie sich durchflechten und ein dünnes zartes Netz bilden. Diese schon von Weigert bei der normalen Neuroglia des Menschen beobachtete Erscheinung findet auch bei der pathologischen Neuroglia-Bildung statt.

$\mathrm{Zu}$ den wichtigsten Entwickelungs-Eigenschaften der pathologischen Neuroglia sind, m. E., diejenigen zu zählen, welche sich auf die topographischen Verhältnisse beziehen.

Beim ersten Blick konnte man die Annahme für richtig halten, dass die meisten Nouroglia- und deshalb auch FäserchenBildungen sich rings um die alten Zerstörungsheerde der Nervensubstanz finden, dass man also um alte apoplektische Heerde 
(Cysten), um Tuberkelknötchen oder um Parasiten eine NeurogliaVerdichtung anstatt der zu Grunde gegangenen Nervensubstanz finden könnte. Die histologische Untersuchung bestätigt aber diese Annahme in Wirklichkeit nicht. Es scheint mir das ein sicherer Beweis dafür zu sein, dass die Wirkung des Fremdkörpers (Parasiten) oder der pathologischen Producte (extravasirtes Blut, Transsudate, Zersetzungs-Producte, pathologische Producte, Tuberkel, Endotheliom u. s. w.) nicht geniigt, um die Neubildung der Neuroglia-Zellen und -Fäserchen anzuregen, sondern dass dazu noch andere Bedingungen nöthig sind. Die erste von diesen ist mit grosser Wahrscheinlichkeit die Rückkehr der Neurogliazellen zum embryonalen Zustand, und da solche Rückkehr nicht sehr leicht stattfindet, darf man woll vermuthen, dass dies nur durch einige Zellen-Uebergänge und Erzeugungen geschieht. Die Bildung von Fäserchen folgt, wie oben erwähnt, der ZellEntwickelung; man darf aber nicht sagen, dass diese Bildung der Zellenzahl immer entsprechend sei. Aus den ziemlich zahlreichen, von mir untersuchten Gliomen geht thatsächlich hervor, dass die Bildung von Fäserchen manchmal die Zahl der Zellen ausserordentlich überragt, während ich ein anderes Mal genau das Entgegengesetzte constatirt habe.

Wenn beim zweiten Falle eine Erklärung möglich ist durch die Annahme, dass die Neurogliazellen einen gewissen Entwickelungsgrad erreichen, bevor sie zur Fäserchen-Bildung geeignet sind, so ist es zur Erklärung des entgegengesetzten Vorganges, der überwiegenden Bildung von Fäserchen gegenüber den Zellen, nothwendig, sich folgende zwei Möglichkeiten vorzustellen:

a) Die Fäserchen kännnen auch aus der intercellularen Substanz entstehen, indem ilıre Differenzirung aus einem Material stattfände, welches einige vom Protoplasma abstammende Producte enthalten kann.

b) Nachdem die Neurogliazellen viele Fäserchen gebildet haben, erschöpfen sie sich und gehen zu Grunde.

Die erste Möglichkeit habe ich schon oben discutirt, es ist mir jedoch unmöglich, über die zweite mich zu äussern, welche noch der Erforsehung harrt. 
Bezüglich der topographischen Anordnung der Fäserchen bei dem pathologischen Neuroglia-Gewebe muss ich hier erwähnen, dass dieselbe grossen Veränderungen unterworfen ist und sich meistens innerhalb der Grenze der Nervensubstanz hält; es geschieht aber auch in einigen Fällen, dass sie diese Grenzen überschreitet und direct in Verbindung mit dem Bindegewebe der Pia Mater tritt oder in die Centralhöhlen eindringt, je nachdem die gliomatöse Neubildung sich an der Hirn-Oberfläche oder an dem Mittelhirne oder an dem Kleinhirne entwickelte, oder tief, näher den Centralhöhlen, entsteht. Im ersten Falle durchdringt die Masse der netzartigen Fäserchen nach und nach die Pia Mater und geht mit der Adventitia der Blutgefässchen und mit der Capillarwand eine so innige Verbindung ein, dass es nicht mehr möglich ist, die Grenze zwischen dem NeurogliaGewebe und dem Bindegewebe zu bestimmen, und dass schliesslich die Trabekel des letzteren die Stütze bilden, auf welcher die neuen Neuroglia-Fasern sich ausdehnen.

Im zweiten Falle dagegen ist die Ausdehnung der Neubildung beschränkter uud stellt sich als kleine Knoten oder flache, rundliche Erhebungen dar, welche auf das Ependym gefallenen Wachstropfen vergleichbar sind.

Einen Fall von diesen Neubildungen konnte ich vor 2 Jahren bei einem 32 Jahre alten Manne beobachten. An dem Ependym des lateralen linken Ventrikels erschienen Knoten, welche bald isolirt und linsenförmig, bald so vereinigt waren, dass sie, besonders an der Oberfläche des Septum lucidum, eine pilzartige Masse bildeten.

Diese Knötchen und diese pilzförmigen Massen waren ausschliesslich aus runden; kleinen, mit einem einzigen, leicht färbbarem Kerne und mit geringem, durchsichtigem und unfärbbarem Protoplasma versehenen Zellen gebildet. Diese Zellen von embryonalem Typus waren so dicht aneinander gedrängt, dass man kaum Grundsubstanz und keine Fäserchen zwischen denselben bemerkte.

Im Gegentheil konnte man eine reichliche Neubildung von Neuroglia-Fäserchen beobachten, nehmlich zwischen den proliferirten Ependymzellen und der umgebenden nervösen Substanz. 
In diesem Falle hatte die Neubildung mehr die histologischen Eigenschaften des Sarcoms, als diejenigen des ächten Glioms, vornehmlich wegen der geringen Bildung von Fäserchen, welche wahrscheinlich deshalb so gering war, weil die mehr an der Oberfläche gelegenen Zellen einen embryonalen Typus bewahrten und deshalb nicht die Eigenschaft besassen, Fäserchen zu. bilden.

Dieser Befund von centralem, ependymalem und subependymalem Gliom beweist mit grosser Deutichkeit, dass das Aggregat von Embryonalzellen, aus denen die Fäserchen später entstehen, sich von dem Sarcom-Gewebe in keiner Weise histologisch unterscheidet. Dasselbe ist von den Sarcomen nur dadurch unterscheidbar, dass die mehr peripherischen Zellen die Neigung zeigen, sich za verringern, indem sie eine intercellulare Substanz zwischen sich aufnehmen, in der viele Neuroglia-Fäserchen sich differenziren.

Ausser diesem Befunde aber, welcher zweifellos beweist, dass die pathologische Neuroglia-Bildung sich wie ein Bindegewebe verhält, giebt es einen anderen Befund, der uns, wenn er auch in Bezug auf seine morphologischen Charaktere durchaus verschieden ist, zu demselben Schluss führt, und sich auf die Entwicklung von Fäserchen an der Oberfläche der Nervencentren und auf ihre Verbreitung in der Pia Mater durch excentrische Ausstrahlung bezieht. Der sehr interessante Fall war eine ausgedehnte Gliose der Vierhügel, der oberen Kleinhirnschenkel, des obersten Theiles des Wurmes, welche ich bei einem 10 Jahre alten Mädchen fand (Galliolo).

Trotz der beträchtlichen Neuroglia-Neubildung am frischen Material bemerkte man keine Form- und Färbungs-Veränderung an den oben erwähnten Theilen; man constatirte nur, dass ihre Festigkeit viel grösser geworden wal.

Bei den nach der Weigert'schen Methode behandelten Präparaten konnte man eine merkwürdige Atrophie und einen Schwund der Nerven-Elemente feststellen; an ihrer Stelle fand sich ein dicht gedrängtes Neuroglia-Fasernetz, in dessen Mitte kleine, runde oder spindelförmige Zellen entbalten waren. Neben diesen Neurogliazellen bemerkt man grössere, mit grossen Protoplasma-Fortsätzen, gut differenzirten Kernen und grossen Kör- 
perchen versehene Zellen. Nach ihrem Verhalten gegenüber der Weigert'schen Methode und nach anderen morphologischen Eigenschaften konnte man solche Zellen als übergebliebene Ganglienzellen, aber nicht als neugebildete nervöse Elemente betrachten.

Aber der wichtigste Befund bezieht sich auf die gliomatöse Neubildung an den Kleinhirnwindungen, an den höchsten Theilen des Wurmes und der lateralen Massen. Schon bei schwacher Vergrösserung bemerkt man, dass die Form der Windungen unverändert geblieben ist; die Windungen sehen aber atrophisch und verlängert aus, wie durch einen nach lateraler Richtung von einer zwischen den Windungen befindlichen Neubildung von Fäserchen geübten Drack (Taf. XVa Fig. 3.)

In der Molecularschicht erscheint ein System von dicht gedrängten Fäserchen, welche sich strahlenförmig von aussen nach innen, von der Oberfläche nach der Tiefe verlaufen.

Diese radial gerichteten Fäserchen, von denen einige sehr zart und dünn, andere verhältnissmässig gross sind, verlaufen neben dem oberflächlicheren Theile der molecularen Scbicht nach horizontaler Richtung und bilden damit eine dichtere Schicht. In der Tiefe ziehen sich dieselben Fäserchen um die Purkinje'schen Zellen herum, dringen durch die granulirte Schicht und treten in Beziehung zu den dichten Fäserchen, welche sich durch die markhaltige Substanz der Länge nach verbreiten. Die Bündel der strahlförmigen Fasern der molecularen Schicht entsprechen den sogenannten Bergmann-Deiters'schen Fäsorchen, sie sind aber wegen der Gliose bedentend vermehrt.

In einem pathologischen Proliferations-Zustand finden sich ebenso die Fäserchen, welche bei meinen Präparaten deutlich schwarz-blau gefärbt erscheinen, an den tiefsten Stellen der molecularen Schicht und an der granulirten Schicht; denn es ist, namentlich nach den Weigert'schen Versuchen, bekannt, dass die Neuroglia-Fasern an diesen Punkten sehr spärlich sind. Die Purkinje'schen Zellen sind atrophisch, ihre Zahl ist geringer geworden, ihre Fortsätze sind kurz, und um dieselben herum beobachtet man oft Fäserchen, die bogenartig liegen und eine Art von Nische bilden.

Die Marksubstanz zeigt eine starke Verdichtung von Fäser- 
chen, die sich der Länge nach verbreiten, sie sind zahlreicher im-Centrum, als an der Peripherie, d. h., neben der granulirten Schicht, wo sie sich mit den strahlenförmigen Deitersschen Fäserchen durchflechten (Taf. XV Fig. 3). Diese strahlenförmigen Fäserchen von Bergmann-Deiters bleiben aber nicht auf die moleculare Schicht in ihrer ganzen Ausdehnung während dieser pathologischen Vermehrung beschränkt, sondern sie treten an vielen Punkten aus dieser Sehicht heraus, indem sie Faserbündel bilden, welche nach derselben strahligen Richtung sich längs einer kurzen Strecke verbreiten, und sich dann auflösen in einer Masse von dünnen Fäserchen, aus welcher eine netzartige, mit sehr dichten Maschen versehene Bildung entsteht, welche zwischen den Lappen der Kleinhirn-Windungen liegt (Taf. XVa Fig. 3).

Diese netzantige Masse, welche nichts Anderes, als eine Emanation der Bergmann-Deiters'schen Fasern darstellt, besteht aus Neuroglia-Fäserchen. Im Inneren derselben sind neugebildete Blutgefässe enthalten, um welche die Fäserchen sich verdichten und unregelmässige Zonen vorbanden sind, die die Fäserchenbündel auf verschiedene Weise scheiden.

Die innerhalb dieses dichten und zarten Fasernetzes befindlichen Zellen sind meistens klein, rund und entsprechen den sogenannten Astrocyten.

Die bis jetzt beschriebene Läsion, welche ich $\mathbf{n}$ beobachten hatte, ist verhältnissmässig nicht sehr weit vorgeschritten, und gerade deshalb ist es uns möglich, die Anordnung der neu gebilcleten Fasern bezüglich der normalen Lage der Fäserchen in der Kleinhirn-Rinde, in der granulirten Schicht und in der markhaltigen Substanz richtig aufzufassen. Viel weiter vorgeschritten aber ist die von der Neuroglia-Neubildung hervorgebrachte Veränderung an den oberen Theilen des Wurmes. Die Windungen sind meist zu Grunde gegangen und zerstört, die moleculare Schicht ist verschwunden, die Purkinje'schen Zellen und die granulirte Schicht sind ebenso zerstört, das Ganze ist durch eine unregelmässige Masse von Neuroglia-Fäserchen ersetzt, welche, bald gross, bald dünn, nach verschiedener Richtung gehen und ein dichtes Netz bilden.

An dem oberflächlichen Theil dieser Neuroglia-Anhäufung, 
welche die Neigung hat, sich excentrisch zu verbreiten, bemerkt man papillenförmige, von Fäserchen-Pinseln gebildete und strahlenförmige Hervorragungen. Solche Hervorragungen sind in kleineren Zwischenräumen verbunden durch andere, quer gerichtete Fäserchen. Ohne Zweifel hat diese grosse Masse von neugebildeter Neuroglia sich allmählich entwickelt, je nachdem der Zerfall der Kleinhirn-Substanz stattfand. Somit hat sie sichwie ächtes Bindegewebe verhalten.

Der eben kurz berichtete histologische Befund ist neu und auch sehr seltsam, da die Neuroglia-Neubildung ausserhalb der molecularen Kleinhirn-Schicht viel grösser ist, als innerhalb derselben. Das hängt wahrscheinlich von dem geringeren Widerstand bei der Fäserchen-Entwicklung und von der.Stütze ab, welche die zarten bindegewebigen Lamellen der Pia mater den Fasern während ihrer Entwicklung gegeben haben. Solche bindegewebigen Lamellen sind innerhalb des dichten Neuroglia-Netzes nicht mehr bemerkbar.

Unter anderen, die Anordnung der neugebildeten NeurogliaFäserchen betreffenden Eigenschaften müssen wir die auf das Verhältniss zwischen den Fäserchen und den Blutgefässen sich beziehenden erwähnen.

Im Allgemeinen sind die Blutgefässe von einem Mantel von Fäserchen umgeben, die sich in doppelter Sohicht anordnen: in einer änsseren, in welcher dje Fäserchen der Länge nach oder parallel der Richtung des Gefässes verlaufen, und in einer inneren, welche aus ringförmig angeordneten Fäserchen besteht. (Taf. XV Fig. 4).

Manchmal sind diese Schichten nicht, gut individualisirt, und in diesem Falle bemerkt man der Länge nach oder quer: gerichtete Fäserchen, welche den Neuroglia-Mantel der Gefässe bilden.

Wäbrend es um die grösseren Gefässe herum einen Raum zwischen Adventitia und Neuroglia-Neubildung giebt, fehlt ein solcher Raum um die Capillaren, und die Fäserchen stützen sich unmittelbar auf die Capillarmembran.

Diese Anordnung beobachtet man namentlich bei den Gliosen und bei den harten Gliomen, wo die Zellen spärlich sind. 
Eine andere Eigenschaft der Fäserchen in Bezug auf ihre Topographie ist das Verschwinden der Verdichtungszonen bei einigen Gliomen. Solche Verdichtungszonen stimmen meist mit der numerischen Verminderung, oder mit dem Verschwinden der zelligen Gliom-Elemente überein (Taf. XV Fig. 1).

Endlich ist noch eine andere Eigenschaft zu erwähnen, nehmlich das Verhalten der Fäserchen bei den Gliomen der Hirnrinde.

In der oberflächlichen Schicht, d. h., bei der sogenannten molecularen Schicht, sind die Fäserchen sehr dünn, dicht gedrängt und verlaufen meist fast parallel der Oberfläche der Schicht selbst; zwischen ihnen erscheint eine fein granulirte Substanz, innerhalb deren wenige Zellelemente sich befinden. In den tiefen Schichten dagegen, wo Neurogliazellen von verschiedenem morphologischem Typus im Ueberfluss vorhanden sind, ist die Fäserchen-Vertheilung unregelmässig, wie bei den centralen Gliomen.

Aus den oben berichteten histologischen Befunden geht nun hervor, dass man, so veränderlich auch die Fäserchen-Anordnung bei der pathologisch gebildeten Neuroglia ist, entsprechen den verschiedenen Typen der für die Neuroglia charakteristischen Zellelemente, sich doch mehr und mehr überzeugt, dass die Neuroglia in pathologischen Zuständen, Eigenschaften annimmt, welche sie mehr dem Bindegewebe als dem Epithelgewebe nähern.

Man muss aber nicht vergessen, dass dieselbe eine EctodermBildung ist, d. i. ein Epithelgewebe, welches für seine besondere Function, die Nervenfasern, die Ganglienzellen und auch die Blutgefässe zu stützen, seine morphologischen Charaktere so tief verändert hat, dass es mehr einem Bindegewebe, als einem Epithel gleicht.

Diese Thatsache nun, welche schon von Anderen beobachtet worden ist und von den meisten Histologen angenommen wird, bestätigt sich auch in pathologischen Zuständen, wo sie sich sogar deutlicher sich zeigt.

Während des Zustandes der pathologischen Neubildung zeigen sich aber, wie dies aus meinen oben erläuterten Präparaten hervorgeht, einige wesentlichen Eigenschaften der verschie- 
denen Neuroglia-Elemente, welche aufs Neue die ectodermische Entstehung derselben bestätigen würden.

Das wird thatsächlich durch dasVorhandensein vonEpithel-Inseln oder Resten des ursprünglichen Neuroepithels innerhalb gliomatöser Neubildungen bewiesen. Ebenso durch das epitheliale Aussehen der von mir so genannten gliogenetischen Zellen, welche eine Rückkehr zu dem Embryonalzustande der Gliazellen darstellen würden. Das wird auch durch die GliazellenAnordnung bei gewissen Gliomen und ihr Verhältniss zu den Fäserchen dargethan. Es giebt also Eigenschaften, durch welche das Neuroglia-Gewebe sich von dem Bindegewebe und von anderen pathologischen Producten unterscheidet, wie auch Eigenschaften bemerkbar sind, durch welche das Neuroglia-Gewebe mehr dem Bindegewebe gleicht.

Eine Aehnlichkeit dagegen mit den Epithelgeweben fehlt ganz und gar der normalen. Neuroglia erwachsener Menschen; sie erscheint nur in bestimmten Phasen der pathologischen Neuroglia-Bildung, sobald die Zellelemente ihre EmbryonalCharaktere wieder annehmen.

Der Unterschied von dem Bindegewebe ist am meisten ein chemischer, kein morphologischer. Kalilösungen zerstören allmählich die Neuroglia-Fäserchen, indem sie dieselben erweichen, aber nicht das Bindegewebe. Anhäufungen neugebildeter Neuroglia gehen nicht so leicht, wie Bindegewebe, regressiven Metamorphosen (Sklerosis, Verkalkung) entgegen, sondern sie erweichen leichter und sind dem granulirten Zerfall unterworfen wegen des Oedems oder der Nekrobiose des neugebildeten Neuroglia-Gewebes.

Das Neuroglia-Fäserchennetz unterscheidet sich von Fibrin erstens dadurch, dass die einzelnen Fibrin-Fasern stärker und kürzer sind, sich verzweigen und anatomosiren, indem sie kleine Räume umgrenzen, innerhalb deren Reste von rothen und weissen Blutkörperchen enthalten sind; zweitens dadurch; dass die FibrinTrabekeln nicht, wie die Neuroglia-Fäserchen, den granulirten Zerfall erleiden.

Die Aehnlichkeit dagegen zwischen Neuroglia und Bindegewebe zeigt sich andererseits nicht nur im normalen Zustande, insofern als die Neuroglia sich bei Erwachsenen wie ächtes Stätzgewebe verhält, sondern auch bei der pathologischen Neu- 
bildung, namentlioh durch die grosse Neigung, sich an Stelle der zu Grunde gegangenen nervösen Elemente zu setzen, ferner durch die Möglichkeit, das perinervöse Bindegewebe (Pia mater), die Gefäss-Adventitia oder die Wände der Capillaren und die lymphatischen Räume zu durchdringen, und endlich durch die Weise, in -welcher die Neurogliazellen sich manchmal vereinigen, indem sie den embryonalen Typus festhalten und sich wie sarcomatöse Zellen anordnen. Das Verhalten der Neuroglia bei der pathologischen Neubildung ist dem der entzündlichen Neubildung von Bindegewe be ähnlich. Aber erstere unterscheidet sich von letzterer erstens dadurch, dass eine offenbare wirkliche Reizung fehlt, denu wir beobachten im Allgemeinen keine oder sehr pärliche NeurogliaBildung um Parasiten, solitäre Twrberke], hämorrhagische Heerde u. s. w., wie man sie gewöhnlich bei analogen Läsionen des Bindegewebes bemerkt, zweitens dadurch, dass eine solche Neuroglia-Neubildung viel mehr atypisch ist, d. h., mit dem Verschwinden von Elementen ïbereinstimmt, welche einen mehr. hervortretenden und länger dauernden Embryonaltypus bewahren.

\section{Erklärung der Abbildungen auf Tafel XIII-XV.}

T a f. XIII, Fig. 1 u.2. Grosse, mit epithelialen Elementen ausgekleidete Räume oder Spalten in einem Gliom des linken Gyrus uncinatus und der subcorticalen weissen Substanz des Scheitellappens bei einem 6 jährigen Kinde. In Fig. 1 ist die oberflächliche Strecke einer solchen Spalte dargestellt. - Die Epithelzellen bilden eine vollständige Auskleidung der inneren Oberfläche dieses Raumes. Solche Epithelbildungen aa stellen Reste des Neuroepithels dar: bb dichtes Neuroglia-Fasernetz um die epitheliale Bildung herum cc. Kleine, rundliche Neurogliazellen. Färbung nach Weigert'scher Methode.

Fig. 2. An diesem Präparate bemerkt man, dass die epithelialen Elemente nicht nur eine Auskleidung der Wände einer unregelmässigen Höhle bilden (a), sondern auch in das dicke Glia-Fasernetz sich verbreiten, indem sie eine Art von Epithelialzellen-Schnur bilden, welche von Neuroglia-Fasern (b) umgeben ist. - Auf der rechten Seite der Abbildung sieht man einige dieser epithelialen Zellen (cc) innerhalb des Gliom-Netzes isolirt, ohne dass ein Raum zwischen ihnen und dem Reticulum bestebt. Andere von diesen Zellen sind in Spalten eingeschlossen.

Fig. 3. Zwei von diesen atypisehen Epithelzellen in starker Vergrosserung inmitten des neugebildeten Neuroglia-Fasernetzes. 


\section{6}

Fig. 4. Grosse, ründliche, epithelartige, innerhalb einer gliomatösen Neubildung eingelagerte Zelle (gliogenetische Zelle). bb. Runde oder ovale Zelle der neugebildeten Glia. Färbung nach Weigert'scher Methode.

Fig. 5. aaa. Gliogenische, abgeplattete, unregelmässige, grosse Zellen. bb. Kleine gliogenische Zellen.

Taf. XIV, Fig. 1. Schnitt durch ein junges Knötchen von einem multiplen Gliom der Hirnrinde bei einem 22 jährigen Manne (Agostini). aa. Dicbtes Neuroglia-Fasernetz. bbb. Gliogenische, innerhalb des Fasernetzes vertheilte oder in kleinen Räumen eingenistete Zellen. c. Thine Reihe von ovalen, durch directe Theilung gliogenischer Zellen entstandenen Kernen, ähnlich der Kernschnur einer in Regeneration befindlichen quergestreiften Muskelfaser.

Fig. 2. Schnitt aus einem weichen Gliom des Thalamus opticus bei einem 16 jährigen Mädehen (LazZaro). a. Grosse gliogenische, in Ruhelage gesehene Zelle. bb. Kleine, runde Neurogliazellen. c. Sprossende Kernmasse, welche die Vermebrungsweise der gliogenischen Zellen zeigt.

Fig. 3. Schnitt eines Glioms des Centrum ovale bei einem Erwachsenen. Grosse, dreieckige, unregelmässige, mit blau gefärbtem Protoplasma und mit grossen protoplasmatischen Fortsätzen versehene Zellen.

Fig. 4. Schnitt durch den tiefsten Theil eines Glioms der 1. u. 2. linken Frontal-Windung und der weissen subcorticalen Substanz (Baessato). aa. Faserbündel, welche, indem sie sich gegenseitig kreuzen, einige Räume umgrenzen, innerbalb deren grosse, ovale Zellen enthalten sind. bb. Zellen mit hyalinem, nach der W eig er tschen Methode nichtfärbbarem Protoplasma. cc. Eine Blutcapillare, auf deren Wänden die neugebildeten Neuroglia-Füserchen aufsitzen.

Fig. 5. Schnitt durch den oberflächlichen Theil desselben Glioms. aa.Grosse, unregelmässige, mit einem, selten mit zwei Kernen versehene Zellen von epithelialem Ausseben. bb. Neuroglia-Fäserchen, die sich dicht an die erwähnten Zellen drängen.

Fig. 6. Sebnitt durch ein Gliom des linken Scheitellappens bei einem 6 jährigen Kinde. Mittelgrosse, unregelmässige, mit schwach färbbarem Protoplasma und mit ziemlich kurzen, protoplasmatischen Ausläufern versehene Zelle, welche von tiefblau gefärbten Neuroglia-Fasern durebkreuzt ist.

Taf. XV, Fig. 1. Schnitt durch ein Gliom des rechten Frontallappens bei einem Erwachsenen. aa. Grosse gliogenische Zellen. b. Sprossende Kernmasse. c. Vedichtungszone der Neuroglia-Fasern mit Schwund der zelligen Elemente. dd. Pericelluläres Fasernetz. 
Fig. 2. Schnitt eines Glioms des Balkens und der Inselwindungen. A. Junger Theil des Glioms, welcher an zelligen Elementen reich ist. Diese sind in einer $Z$ wischensubstanz zerstreut, wo die Fäserchen fehlen. b. Alter Gliom-Theil, wo die zelligen Elemente weniger zahlreich sind und die Zwischensubstanz reich an Fäserchen ist.

Fig. 3. Ausgedehnte Gliose der Vierhügel, der oberen Bindearme und des oberen Theils des Wurmes bei einem 10 jährigen Mädchen. Schnitt durch eine Kleinhirn-Windung beim Lobus paracentralis, wo die gliomatöse Neubildung noch nicht so vorgerückt ist, um die Kleinhirn-Substanz vollständig zu ersetzen. aa. Molecularschicht, in weleher die strablenförmigen Fasern (BergmannDeiters'sche Fasern) sebr zahlreich und deutlich sind. bb. Starke Neuroglia-Bildung $z$ wischen den Kleinhirn-Windungen. cc. Bergmann's strahlenförmige Fasern, welche von der Molecularschicht in die extranervöse Neuroglia-Bildung gehen, wo sie sich zerstreuen, indem sie die Richtung wechseln. dd. Granulirte Schicht der Kleinhirn-Rinde. ee. Purckinje'sche Zellen, die in der Zahl bedeutend vermindert und atrophisch geworden sind. f. Faserreiche Marksubstanz.

Fig. 4. Anordnung der Făserchen der neugebildeten Neuroglia um die neuen Blutgefässe herum. aa. Longitudinale Fasern. bb. Quergerichtete und ringförmige Fasern. c. Blutcapillare, deren Wände mit ovalen Kernen versehen sind.

\section{XXVI. \\ Die Heilung der Epidermis \\ von \\ Dr. med. Heinrich von Bardeleben,}

d. Z. Volontär-Assistenten am Pathologischen Institut zu Berlin.

(Hierzu 13 Text-Abbildungen.)

I.

Die Thatsache, dass kleinere Lücken, die die Oberhaut durchsetzen, sich von selbst ergänzen, ist allbekannt und gilt jedem Laien als selbstverständlich. Die feineren Vorgänge hierbei sind jedoch nicht so einfach, wie es danach den Anschein haben möchte, sondern so verwickelte, dass sie zu den widersprechend- 